Noname manuscript No.

(will be inserted by the editor)

\title{
Interplay between turbulence and waves: Large-scale helical transfer, and small-scale dissipation and mixing in fluid and Hall-MHD turbulence
}

\author{
Annick Pouquet • Duane Rosenberg • \\ Julia E. Stawarz
}

Received: date / Accepted: date

\begin{abstract}
Novel features of turbulent flows have been analyzed recently, for example: (i) the possibility of an ideal invariant, such as the energy, to be transferred both to the small scales and to the large scales, in each case with a constant flux; (ii) the existence of non-Gaussian wings in Probability Distribution Functions of kinetic, magnetic and temperature fluctuations, together with their gradients, thus displaying large-scale as well as small-scale intermittency; and (iii) the linear dependence on the control parameter of the effective dissipation in turbulence when non-linear eddies and waves interact. We shall briefly review these results with examples stemming from Solar Wind data, the atmosphere and the ocean with either magnetic fields, stratification and/or rotation. In a second part, we shall examine numerically the inverse cascades of magnetic and of generalized helicity for Hall MHD in the presence of forcing. These helical invariants in the ideal non-dissipative case involve various cross-correlations between the velocity and vorticity, the magnetic field and the magnetic potential. For an ion inertial length larger than the forcing scale, the effect of the waves is significant. It leads to an exponential attenuation of the inverse cascade to large scales, since, through the velocity and vorticity, small scales play an increasing dynamical role for a strong Hall current.
\end{abstract}

Keywords Turbulence · Waves · Hall-MHD · Helicity · Inverse Cascades

PACS 47.27.Jv · 47.35.-Jk $\cdot 47.51 .+\mathrm{a} \cdot 47.65 . \mathrm{d} \cdot$ 92.10.Lq $\cdot 94.05 . \mathrm{Lk}$

JES is supported by UKR/STFC grant ST/S000364/1

A. Pouquet

Laboratory for Atmospheric and Space Physics, University of Colorado, Boulder, CO 80309; and National Center for Atmospheric Research, P.O. Box 3000, Boulder, CO 80307, USA. Tel.: +1-303-4971860 E-mail: pouquet@ucar.edu.

D. Rosenberg

288 Harper St. Louisville, CO, 80027 USA.

J. Stawarz

Department of Physics, Imperial College London, United Kingdom. 
Noname manuscript No.

(will be inserted by the editor)

\title{
Interplay between turbulence and waves: Large-scale helical transfer, and small-scale dissipation and mixing in fluid and Hall-MHD turbulence
}

\author{
Annick Pouquet • Duane Rosenberg • \\ Julia E. Stawarz
}

Received: date / Accepted: date

\begin{abstract}
Novel features of turbulent flows have been analyzed recently, for example: (i) the possibility of an ideal invariant, such as the energy, to be transferred both to the small scales and to the large scales, in each case with a constant flux; (ii) the existence of non-Gaussian wings in Probability Distribution Functions of kinetic, magnetic and temperature fluctuations, together with their gradients, thus displaying large-scale as well as small-scale intermittency; and (iii) the linear dependence on the control parameter of the effective dissipation in turbulence when non-linear eddies and waves interact. We shall briefly review these results with examples stemming from Solar Wind data, the atmosphere and the ocean with either magnetic fields, stratification and/or rotation. In a second part, we shall examine numerically the inverse cascades of magnetic and of generalized helicity for Hall MHD in the presence of forcing. These helical invariants in the ideal non-dissipative case involve various cross-correlations between the velocity and vorticity, the magnetic field and the magnetic potential. For an ion inertial length larger than the forcing scale, the effect of the waves is significant. It leads to an exponential attenuation of the inverse cascade to large scales, since, through the velocity and vorticity, small scales play an increasing dynamical role for a strong Hall current.
\end{abstract}

Keywords Turbulence · Waves · Hall-MHD · Helicity · Inverse Cascades

PACS 47.27.Jv $\cdot 47.35 .-\mathrm{Jk} \cdot 47.51 .+\mathrm{a} \cdot 47.65 . \mathrm{d} \cdot 92.10 . \mathrm{Lq} \cdot 94.05 . \mathrm{Lk}$

JES is supported by UKR/STFC grant ST/S000364/1

A. Pouquet

Laboratory for Atmospheric and Space Physics, University of Colorado, Boulder, CO 80309; and National Center for Atmospheric Research, P.O. Box 3000, Boulder, CO 80307, USA. Tel.: +1-303-4971860 E-mail: pouquet@ucar.edu.

D. Rosenberg

288 Harper St. Louisville, CO, 80027 USA.

J. Stawarz

Department of Physics, Imperial College London, United Kingdom. 


\section{Introduction}

Magnetohydrodynamic (MHD) turbulence differs from ordinary fluid turbulence for several reasons. One of them is the presence of Alfvén waves due to the effect of a uniform, or large-scale, magnetic field, whereas of course a uniform velocity field can be eliminated through a Galilean transformation. This leads to a higher degree of nonlocal interactions, that is interactions between widely separated scales in MHD, and in Hall-MHD in the presence of a Hall current in a generalized Ohm's law [1,2] (see e.g. [3] for nonlocal interactions in the fluid case). The dynamics of conducting flows becomes more complex as one examines smaller scales when plasma effects become prevalent [4]; nevertheless, a description of large-scale events in the cosmos based on MHD has been found to be useful, as reviewed for example in the context of the heliosphere [5-8]. Indeed, most of the energy resides in the largest scales which are controlled and adequately represented by the MHD description in the context of wave-turbulence interactions, as observed in the solar wind [9-11]. However, a large series of observations of the solar wind, magnetotail and magnetosheath using an array of recently-launched satellites (e.g. MMS and Solar Probe) have led to a refined understanding of the role of kinetic scales on the dynamics of this medium [12,13], and in particular of reconnection processes $[11,14,15]$.

Among the many topics tackled by PierLuigi Veltri and his collaborators, the effect of the slowing-down of turbulence transfer to small scales due to Alfvén waves in MHD turbulence $[16,17]$ was developed in a seminal paper in the context of the growth of the cross-correlation between the velocity and magnetic field $\mathbf{v}$ and $\mathbf{b}$ [18], thus explaining the lack of symmetry between the two Elsässer variables $\mathbf{z}^{ \pm}=\mathbf{v} \pm \mathbf{b}$, as observed in the solar wind (see also [19, 20] for more recent data analyses).

A further property of these turbulent flows is the intermittency of smallscale fields such as the current and vorticity, as characterized for example by non-Gaussian wings in their probability distribution functions, for Eulerian and for Lagrangian statistics, as seen in observations [5,21-27] and in numerical simulations [28-32]. These can be studied as well using low-dimensional dynamical systems such as sand-pile models $[33,34]$ that include the properties of self-criticality in solar flares $[35,36]$, or using approaches in the context of reduced-MHD [37]. These small-scale structures lead to anomalous dissipation and reconnection events in two dimensions (2D) [38,39] and in 3D [40-45].

Fluid and MHD turbulence share a lot of properties, determined by the interactions of nonlinear eddies and waves which, for the atmosphere and the oceans, are due to the Coriolis force and gravity (see, e.g. the reviews in [46, $47]$ ). In this context, after giving the governing equations in the next Section, we report in $\S 3$ on three aspects of turbulent flows that have been unraveled recently for both rotating stratified turbulence and for MHD and Hall-MHD. We then move on to describe in a little bit more details the dynamics of magnetic helicity in $\S 4$, and a short conclusion is finally given in $\S 5$. 


\section{Equations and definitions}

We now write for completeness the fundamental equations for the questions we wish to discuss. The Hall-MHD equations in the incompressible case and in the presence of forcing for both the velocity $\mathbf{v}$ and the magnetic field $\mathbf{b}$ (in terms of an Alfvén velocity, with $\mathbf{b}=\mathbf{B} / \sqrt{\mu_{0} \rho_{0}}$ ) are:

$$
\begin{aligned}
& \frac{\partial \mathbf{v}}{\partial t}=-\mathbf{v} \cdot \nabla \mathbf{v}-\nabla P+\mathbf{j} \times \mathbf{b}+\nu \nabla^{2} \mathbf{v}+\mathbf{f}_{v}, \\
& \frac{\partial \mathbf{b}}{\partial t}=\nabla \times(\mathbf{v} \times \mathbf{b})-\epsilon_{H} \nabla \times(\mathbf{j} \times \mathbf{b})+\eta \nabla^{2} \mathbf{b}+\mathbf{f}_{b}, \\
& \quad \nabla \cdot \mathbf{v}=0, \nabla \cdot \mathbf{b}=0 .
\end{aligned}
$$

with $P$ the pressure, $\mathbf{B}, \rho_{0}, \mu_{0}$ the magnetic induction, the constant density and the vacuum permeability; $\mathbf{j}=\nabla \times \mathbf{b}$ is the current density and $\mathbf{a}$ is the magnetic potential, with $\mathbf{b}=\nabla \times \mathbf{a}$. Finally, $\nu$ and $\eta$ are the kinematic viscosity and magnetic diffusivity, and $\mathbf{f}_{v, b}$ are forcing terms, taken here to have random phases. The Hall current is controlled by the dimensionless parameter $\epsilon_{H}=d_{i}$ which is the ion inertial length scale (in terms of the numerical dimension of the box $L_{M}=2 \pi$ ). The MHD equations are recovered for $\epsilon_{H}=0$. In all the results given in this paper, the GHOST code (Geophysical High-Order Suite for Turbulence) was used; it is a solver with spectral accuracy for a variety of fluid and plasma equations. It parallelizes efficiently, including using [48]; it includes the possibility of non-unity aspect ratio, and it now allows for nonperiodic boundary conditions in one direction through a Fourier continuation method [49].

Furthermore, the Boussinesq equations (in the absence of magnetic field), with $\theta$ the temperature fluctuations around a mean profile, and normalized so as to have the physical dimensions of a velocity, are:

$$
\begin{aligned}
\frac{\partial \mathbf{u}}{\partial t}+\boldsymbol{\omega} \times \mathbf{u}+2 \boldsymbol{\Omega} \times \mathbf{u} & =-N \theta \hat{e}_{z}-\nabla \mathcal{P}+\nu \nabla^{2} \mathbf{u} \\
\frac{\partial \theta}{\partial t}+\mathbf{u} \cdot \nabla \theta & =N w+\kappa \nabla^{2} \theta
\end{aligned}
$$

Rotation and stratification are imposed in the vertical $(z)$ direction, $\kappa$ is the diffusivity, and $\boldsymbol{\omega}=\nabla \times \mathbf{u}$ the vorticity; $\Omega$ is the rotation frequency (with $f=2 \Omega$ ), and $N$ is the Brunt-Väisälä frequency.

In Hall MHD, the global ideal invariants (for $\nu=\eta=0$ ) are the total energy $E_{T}=E_{V}+E_{M}=\left\langle|\mathbf{v}|^{2}+|\mathbf{b}|^{2}\right\rangle / 2$, the magnetic helicity $H_{M}=\langle\mathbf{a} \cdot \mathbf{b}\rangle / 2$, and the generalized helicity $H_{G}$ :

$$
H_{G}=H_{M}+2 \epsilon_{H} H_{C}+\epsilon_{H}^{2} H_{V}=H_{M}+\epsilon_{H} H_{X},
$$

with $H_{V}=\langle\mathbf{v} \cdot \boldsymbol{\omega}\rangle / 2$ the kinetic helicity, $H_{C}=\frac{1}{2}\langle\mathbf{v} \cdot \mathbf{b}\rangle$ the cross helicity, and $H_{X}=2 H_{C}+H_{V}, H_{X}$ being also an invariant. For the Boussinesq case, the ideal invariants with $\nu=\kappa=0$, are again the total energy now expressed as $E_{T}^{B}=E_{V}+E_{\theta}=\left\langle|\mathbf{v}|^{2}+\left.\theta\right|^{2}\right\rangle / 2$, and the point-wise potential vorticity 
$P_{V}=f \partial_{z} \theta-N \omega_{z}+\boldsymbol{\omega} \cdot \nabla \theta$. Kinetic helicity is only invariant in the absence of stratification, and is otherwise created by the flow in the rotating stratified case $[50-52]$.

One also defines the relative helicity rates, measuring the alignment or anti-alignment of the vectors involved, through cosine functions, and noting that the terms $\mathbf{h}_{x}, x=a, b$, in the relative rate of generalized helicity are defined as $\mathbf{h}_{a}=\mathbf{a}+\epsilon_{H} \mathbf{v}, \mathbf{h}_{b}=\nabla \times \mathbf{h}_{a}=\mathbf{b}+\epsilon_{H} \boldsymbol{\omega}$ :

$$
\sigma_{M}=\frac{\mathbf{a} \cdot \mathbf{b}}{|\mathbf{a}||\mathbf{b}|}, \sigma_{C}=\frac{\mathbf{v} \cdot \mathbf{b}}{|\mathbf{v}||\mathbf{b}|}, \sigma_{G}=\frac{\mathbf{h}_{a} \cdot \mathbf{h}_{b}}{\left|\mathbf{h}_{a}\right| \mathbf{h}_{b} \mid}, \sigma_{V}=\frac{\mathbf{v} \cdot \boldsymbol{\omega}}{|\mathbf{v}||\boldsymbol{\omega}|} .
$$

Finally, dimensionless parameters measuring the relative strengths of the terms appearing in these equations are written as usual as:

$$
R e=\frac{U L_{i n t}}{\nu}, F r=\frac{U}{L_{i n t} N}, R o=\frac{U}{L_{i n t} f}, R_{B}=R e F r^{2}, R_{I B}=\epsilon_{V} /\left[\nu N^{2}\right]
$$

where $R e, F r, R o$ and $R_{B}$ are the Reynolds, Froude, Rossby and buoyancy Reynolds numbers respectively. $R_{I B}$ is the buoyancy interaction parameter and measures the effective amount of energy dissipation in a stratified turbulence in the presence of waves, properly normalized using the Brunt-Väisälä frequency. $U$ and $L_{i n t}$ are characteristic velocity and length scales for the fluid $\left(L_{\text {int }}\right.$ is called the integral scale), and $\epsilon_{V}=D E_{V} / D T=\nu\left\langle|\boldsymbol{\omega}|^{2}\right\rangle$ is the kinetic energy dissipation rate. The eddy turn-over time $\tau_{N L}$ is defined as $L_{\text {int }} / U$. The Prandtl number $\operatorname{Pr}=\nu / \kappa$ and the magnetic Prandtl number $P_{M}=\nu / \eta$ are assumed to be equal to unity in the following.

For high $R e$, the flow is turbulent and dissipative, whereas for low Fr or low $R o$, the wave periods are faster than the eddy turn-over time characteristic of the nonlinear eddies, and dissipation is less efficient. Note that when $R_{I B}=1$, the Kolmogorov scale $\ell_{D}=2 \pi\left[\nu^{3} / \epsilon_{V}\right]^{1 / 4}$ at which dissipation sets in is equal to the Ozmidov scale $\ell_{O z}=2 \pi\left[\epsilon_{V} / N^{3}\right]^{1 / 2}$ at which isotropy is thought to be recovered (see, e.g., [53]), since one can show that, for a Kolmogorov spectrum at small scale, one has $R_{I B}=\left[\ell_{O z} / \ell_{D}\right]^{4 / 3}$, similarly to $R e=\left[L_{\text {int }} / \ell_{D}\right]^{4 / 3}$ for homogeneous isotropic turbulence.

\section{A few recently-unraveled novel features of turbulent flows}

Although a complete theory of turbulence is still lacking, our understanding of turbulent flows has progressed substantially in this century through the analysis of experimental, observational and numerical data, as well as the use of simplified models. We give below three specific examples.

\subsection{Bi-directional cascades}

A given invariant of the ideal non-dissipative dynamical equations cascades to either small scales (such as the energy in three-dimensional (3D) NavierStokes or MHD turbulence, with a positive flux), or (exclusive) to the large 
scales (such as the energy in 2D Navier-Stokes fluids, or the $\mathcal{L}_{2}$ norm of the magnetic potential in 2D MHD), with a negative flux. But, starting around 2005, data appeared showing fluxes that were, more or less plausibly, constant and with two signs and thus referring to the possibility of two constant-flux cascades, going both to the large scales and to the small scales, starting from the scale at which the energy is injected. This was obtained, for fluid dynamics, for example through a Fourier analysis of ocean drifters [54,55], as well as in laboratory and numerical experiments of strongly rotating flows in the presence or not of stratification [56-59]. It is also observed in three-dimensional fluid dynamics in thin layers, with a clear threshold behavior [60,61]. In MHD, similar detailed analyses were performed on numerical data in two and three space dimensions [62,63], as well as in the solar wind [19], and more recently in the atmosphere of Jupiter [64].

One of the first examples of such a phenomenon, in the case of the Solar Wind using Ulysses data, was observed through the derivation of exact scaling laws [65-67] (see [68] for a recent review); these are linked to the conservation of energy and of cross-correlation in MHD. It was then argued in [19] that this bi-directional cascade was due to the instability of large-scale coherent structures after they form through an inverse cascade which itself is attributed to the quasi bi-dimensionalization of the flow because of the presence of a strong uniform magnetic field.

Another example occurs when taking the case of rotating stratified turbulence (RST), with a forcing at intermediate scales. High-resolution numerical simulations indicated clearly that the direct energy cascade, leading to small-scale mixing and dissipation, was governed by the Froude number, $\mathrm{Fr}$, whereas the inverse energy cascade was governed by the Rossby number, Ro [58] (see also [69]). This is illustrated in the top of Fig. 1 (left), which gives the fluxes in spectral space of potential energy (PE, in blue) and of total (kinetic+potential) energy (in red) for quasi-geostrophic forcing (QG) at $k=10$, that is a forcing with a balance between pressure gradient, Coriolis and buoyancy forces. This was computed for a flow on a grid of $512^{3}$ points and with initial conditions that are also in QG balance. In this case, the initial conditions in the temperature are non-zero, contrary to the runs presented in the other figures. For this run, the governing parameters were $R e \approx 6.8 \times 10^{3}, F r \approx 0.076, R o \approx 0.38, R_{B} \approx 36$ (see [70] for more details).

The PE flux is zero in the large scales, showing that the inverse cascade of total energy, with a constant and negative flux for $k<10$ corresponds to a quasi-two-dimensional flow. The kinetic energy flux (not shown) also displays bi-directional and rather constant ranges. At right are given the corresponding kinetic (KE) and potential (PE) energy spectra for the same flow and at the same time. The kinetic energy displays a clear accumulation in the largest scales for this three-dimensional rotating stratified turbulent flow; strong gradients appear in both fields and correspond to the observed intermittency for flows close to the threshold of instability (see also [53], and below). An embryonic Kolmogorov law $\sim k^{-5 / 3}$ is visible for the kinetic energy for $k \approx 10$ (see indicative line). The potential energy follows the kinetic energy upscale, 
possibly because at this Froude number, inertia-gravity waves are efficient at producing a large-scale potential transfer. At scales noticeably smaller than the forcing, these spectra follow a steep behavior because of the rather low Reynolds number.

\subsection{Large-scale intermittency}

Small-scale eddies in fluid turbulence have been known for a long time to be intermittent, that is highly localized in space and time, and giving rise to non-Gaussian wings in the Probability Distribution Functions (PDFs) of field gradients such as the vorticity [71] and current density [30]. In the ideal case, these events possibly lead to the existence of finite-time singularities as in 2D-MHD [72], or rotational singularities as in 3D MHD [73]. It was shown recently that such non-dissipative flows can nevertheless display turbulent features such as power-law energy spectra, e.g. in a 3D truncated Euler flow [74], as well as in 2D and 3D ideal MHD [75]. In MHD, this quasi-singular behavior, with wings in the PDFs of current and vorticity structures, correspond to topological changes in the geometrical organization of the fields [76] which can be associated with nano-flares emanating from the solar photosphere [22], or Kelvin-Helmoltz instabilities in the magnetosphere [27]. These wings are also observed with Wind data in the Earth's magnetotail [77]; they correspond to the acceleration of high energy electrons, up to $300 \mathrm{keV}$ in reconnection regions, and they display a high degree of anisotropy. It is argued in [78] that this occurs through the reflection of electrons on multiple magnetic islands.

Furthermore, large velocity shear layers can lead as well to intermittent events in the velocity itself in the magnetotail, as argued and shown in [42]. This is akin to what was later observed in stratified turbulence for which anisotropy, intermittency of the vertical velocity and mixing occur in the vicinity (in terms of gradient Richardson number) of the convective and KelvinHelmholtz instabilities [80], an observation also made in the presence of rotation [81, 53,82]; this can be associated with Lagrangian particle acceleration [82]. We show in Fig. 1 (bottom row) two vertical cuts of the temperature fluctuations (left) and of the horizontal velocity (right) for a rotating stratified flow computed on a grid of $4096^{3}$ points in the absence of forcing; the snapshots are given only for a portion of the flow, and are taken at the temporal peak of dissipation. For this run, the Reynolds number is close to 54,000 with a Froude number of 0.024 , a Rossby number of 0.12 and a buoyancy Reynolds number of $R_{B} \approx 32$ (see [79] for details). Note that the stratification of the flow is organized in layers that are slanted because of the rotation, and is a manifestation of the spatial intermittency of the temperature fluctuations. Also note the formation of small-scale structures linked to waves that are convectively destabilized, or for example in the form of Kelvin-Helmoltz billows that further destabilize locally and are one of the sources of both large- and small-scale intermittency and dissipation $[79,81,53]$. 

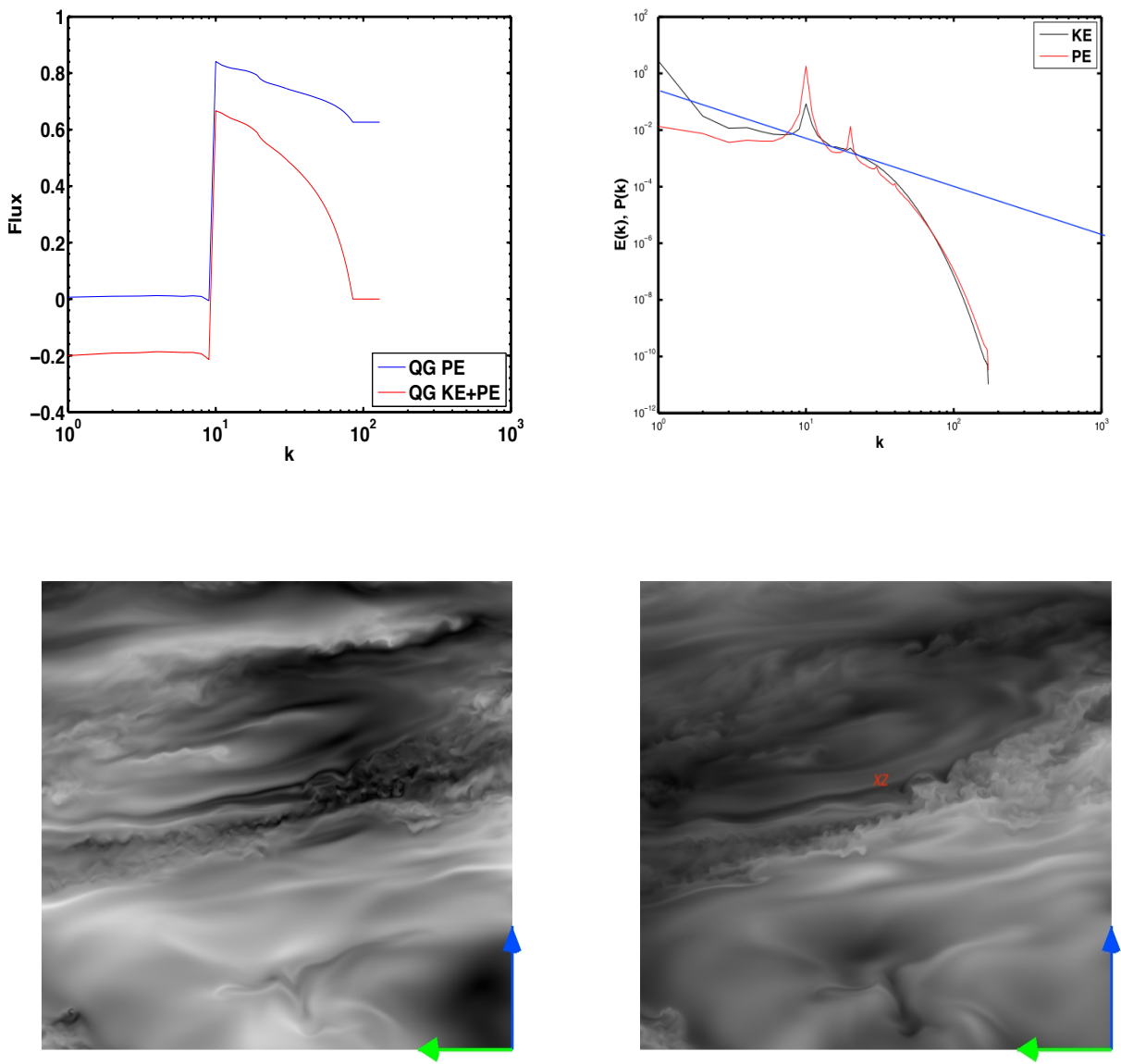

Fig. 1 Top left: Potential (blue) and total (red) energy fluxes (left), and potential (red) and total (black) energy spectra (right). Both plots are for Rotating Stratified Turbulence (RST) for Quasi-Geostrophic (QG) initial conditions; at right, a $k^{-5 / 3}$ line is given for reference [70]. Bottom: Blow-up of a vertical (XZ) cut of temperature (left) and of horizontal velocity (right); grid of $4096^{3}$ points, $R o / F r \approx 5, R e \approx 5.4 \times 10^{4}, R_{B} \approx 32[79]$.

Non-Gaussian wings in the PDFs of the velocity field itself were already obtained and analyzed in numerical simulations of shear flows [83]. They are also observed in the vicinity of the terrestrial foreshock [84], in quantum turbulence in super-fluid Helium [85], and in the computation of normalized third and fourth order moments of temperatures and other synoptic fields in the free troposphere, in the context of re-analysis of climate data [86]. They thus appear as a common feature of turbulence. We note that temporal intermittency is also present in these complex flows.

Moreover, for RST, strong intermittency, as measured by high kurtosis of the vertical velocity and, to a lesser degree, of the temperature fluctuations, 


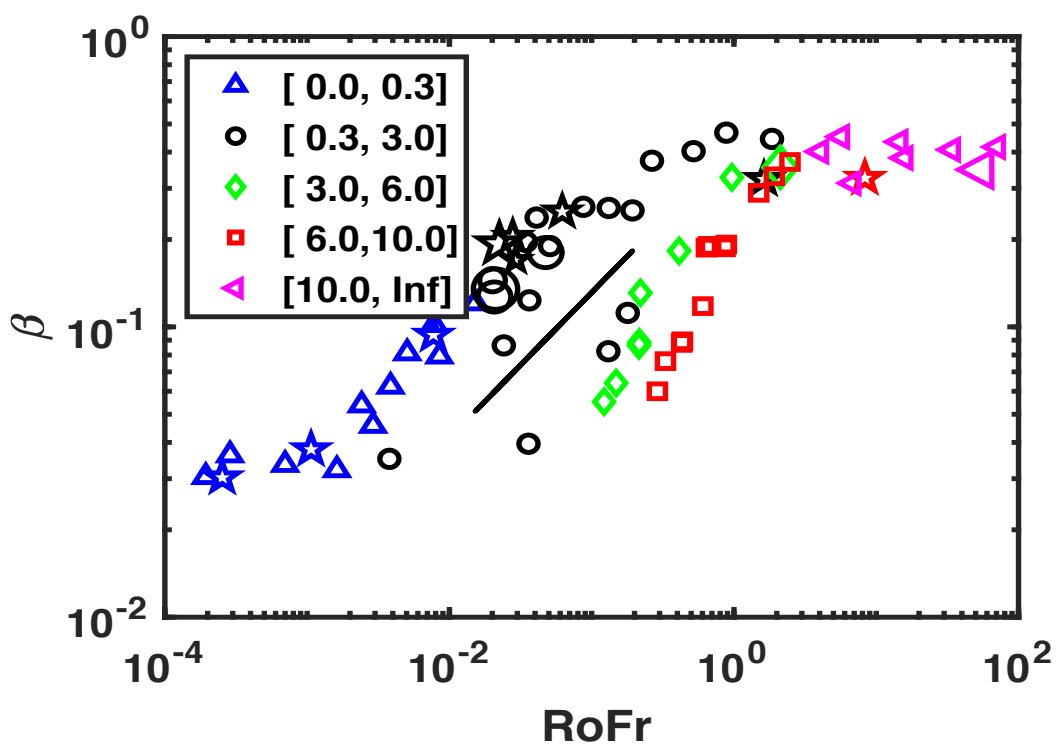

Fig. 2 Efficiency of dissipation as a function of $R o F r$ for a large range of parameters $[70,53]$; color/symbol for Rossby-number intervals are given in insets. A $[R o F r]^{1 / 2}$ scaling, compatible with what is derived in [70], is indicated for reference as a black line.

takes place in a narrow peak of Froude number. This striking behavior can be reproduced following the dynamics of a simple 2-variable system, inspired from the velocity-gradient model of Vieillefosse for strong turbulence [87], in which the linear (wave) terms have been added, as well as dissipation and forcing [81], or in the context of models of the temperature gradient and velocitygradient matrix and its so-called PQR invariants [88,89] ((with $P=0$ in the incompressible case, see [90]).

\subsection{Dissipation efficiency}

As a third example of the significant progress made in our understanding of turbulent flows, and in particular in the presence of waves and anisotropic structures, we now consider the efficiency of dissipation in turbulence. The measured dissipation in fluid and MHD turbulence is known to plateau to a value close to its dimensional expression, namely $\epsilon_{D} \sim U^{3} / L_{\text {int }}[91,92]$ (see also [93]). A recent numerical study in MHD showed that the level at which this efficiency settles depends on the strength of the imposed magnetic field, and thus on the characteristic time scale of Alfvén waves, in a smooth manner [94]. In fact, a simple phenomenological argument can be constructed 


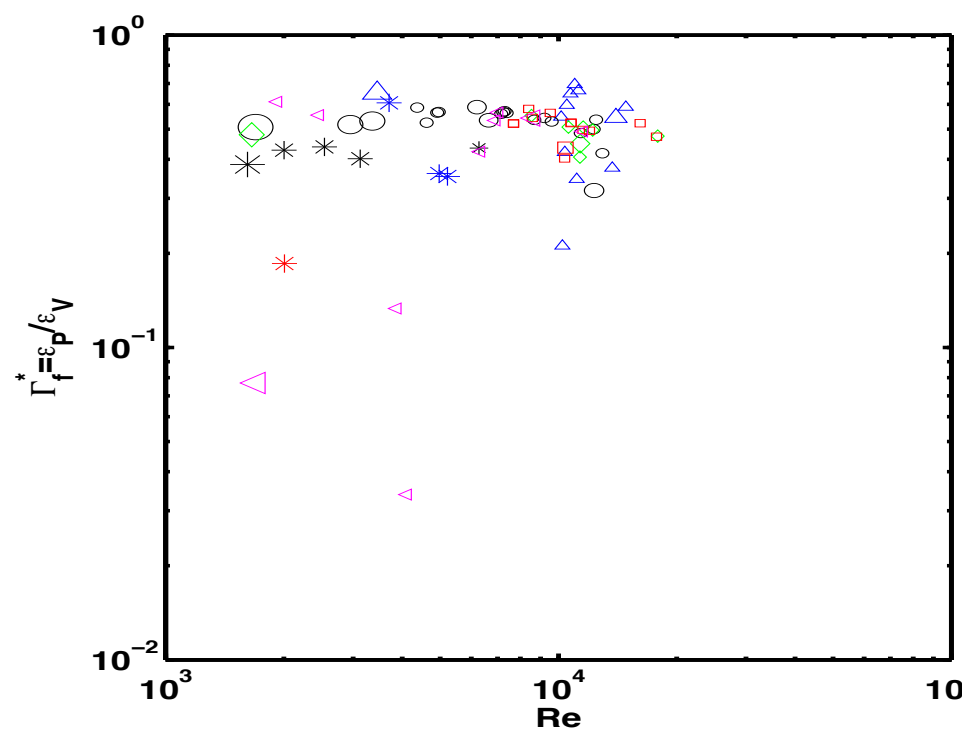

Fig. 3 Efficiency of mixing $\Gamma_{\star}$ defined as $\epsilon_{P} / \epsilon_{V}$ as a function of Reynolds number for the same runs as in Fig. 2.

to show that such a scaling is linear in the control parameter, that is the ratio of the wave period to the nonlinear eddy turn-over time, i.e. the Froude number for stratified flows [70,53]. Such a scaling is compatible, and in fact embodies the slowing-down of energy transfer to small scales because of waveeddy interactions, in a way that is compatible with weak (wave) turbulence.

It was also shown in [95], studying turbulence regimes modified by Alfvén and kinetic Alfvén waves, that the efficiency of (total energy) dissipation is governed as well by the ratio of the turn-over time to the period of the relevant wave, a formulation that includes also the nonlocal effect of a large-scale shear. And a comparison with solar wind observations at electron scales leads to a model of kinetic Alfvén waves for which the dissipation length scale is independent of the energy dissipation rate but depends on the strength of the waves [96]. This is very similar to the case of the so-called saturation regime in the atmosphere and ocean [97] in terms of a balance that is established in the vertical direction between gravity and wave steepening; it leads to a spectrum $E\left(k_{z}\right) \sim N^{2} k_{z}^{-3}$ where the energy injection rate does not appear, and which gives for the dissipation scale in that case $\ell_{\text {diss }} \sim\left[\nu L_{\text {int }} / N\right]^{1 / 3}$, again independent of the energy input $\epsilon_{V}$.

This third novel feature of turbulent flows is illustrated in Fig. 3 and Fig. 4, showing respectively the efficiency of kinetic energy dissipation as a function of $R o F r$, and a measure of small-scale mixing as a function of Re for rotating 

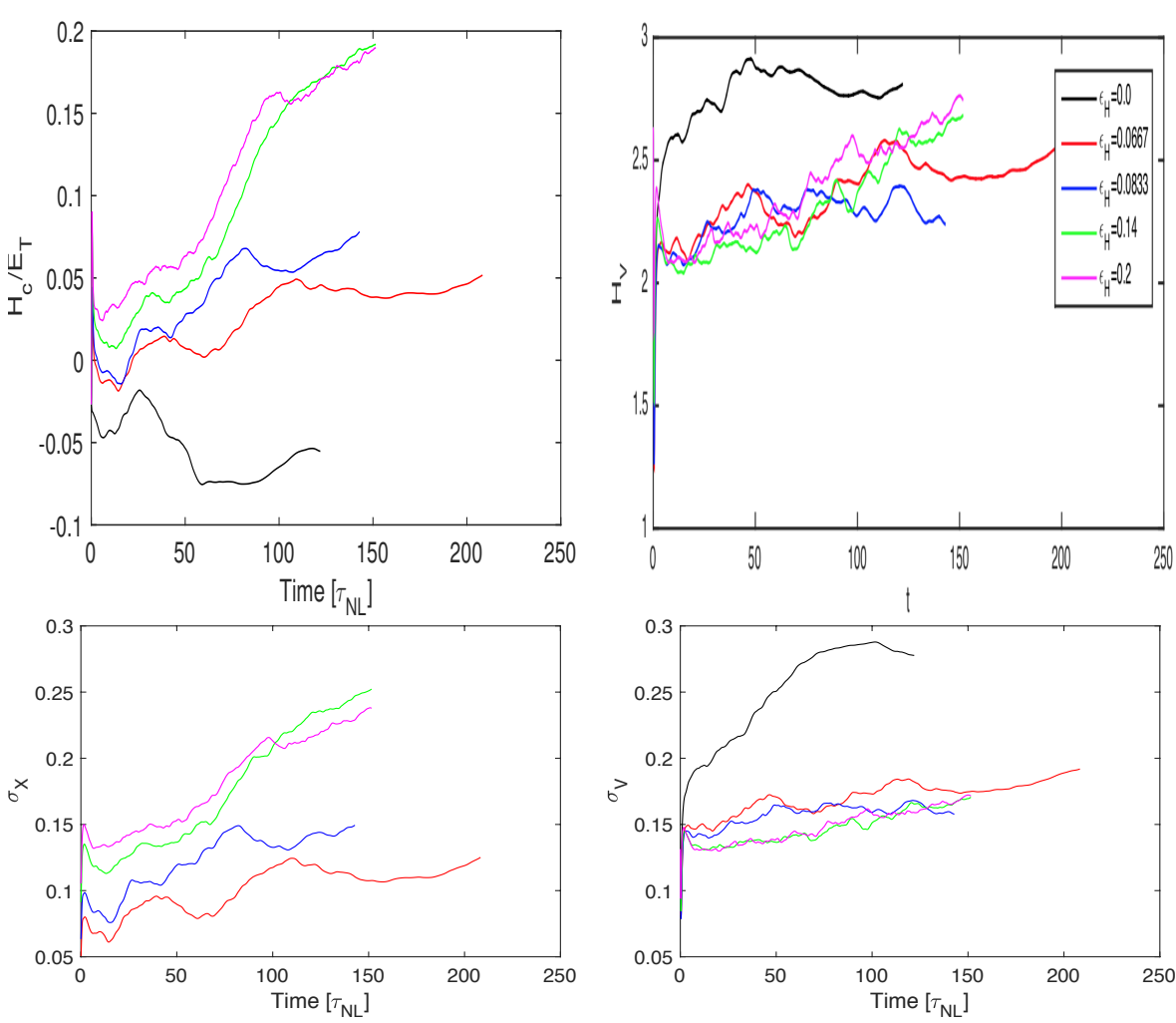

Fig. 4 Top: $H_{C} / E_{T}$ (left) and $H_{V}$ (right) as a function of time for the runs of Table 1 (see inset). Bottom: $\sigma_{X}$ (left) and $\sigma_{V}$ (right) as a function of time. The MHD run is in black.

stratified turbulence. In these scatter plots, stars indicate runs with QG initial conditions, and the size of the symbols is proportional to the viscosity (and inversely proportional to the Reynolds number and numerical resolution).

The measured kinetic energy dissipation $\epsilon_{V}$, normalized by its dimensional evaluation $\epsilon_{D}$ defined above, has been shown to govern the ratio of inverse to direct energy flux [98] in the forced case. Here, the data stems from a large data base of decaying rotating stratified flows [99, 70,53], and the color/symbol scheme, given in the insets, is based on the strength of the imposed rotation. The variation can be seen as following two or three distinct and rather parallel lines, each one close to a $[R o F r]^{1 / 2}$ scaling; this is compatible with the linear scaling in Froude number found previously, provided rotation and stratification play similar roles. Note the shift in data between strongly rotating systems (blue triangles and black circles, at left) and weakly rotating ones (green diamonds, red squares and magenta inverted triangles, at right): stronger rotation, at a given $R o F r$ implies of course a weaker stratification and thus a more turbulent and dissipative flow.

In Figure 3, mixing is defined with the (positive) ratio of potential to kinetic energy dissipation, $\Gamma_{\star}=\epsilon_{P} / \epsilon_{V}$, with $\epsilon_{P}=D E_{P} / D t$. On a logarithmic 
Table 1 List of the runs described in [100] and analyzed further herein, with their identification ID, $\epsilon_{H}$ the Hall parameter and $\sigma_{G}$ the rate of relative generalized helicity. The Reynolds number $R e$ is rather constant and kept at a low value. Finally $k_{d_{i}}=1 / \epsilon_{H}$ is the wavenumber beyond which the Hall term is felt, with a forcing concentrated around $k_{F} \approx 20$. For all runs, $N_{p}=128^{3}$ is the numerical resolution, with $\nu=0.016$ the viscosity; $\sigma_{M}=0.65 ; \sigma_{V}=0.13$ and $\sigma_{C}=-0.027$ are respectively the rates of relative magnetic, kinetic and cross helicities for the forcing (see Equation (7)).

\begin{tabular}{ccccc} 
ID & $\boldsymbol{\epsilon}_{\boldsymbol{H}}$ & $\boldsymbol{\sigma}_{\boldsymbol{G}}$ & $\mathrm{Re}$ & $\boldsymbol{k}_{\boldsymbol{d}_{\boldsymbol{i}}}$ \\
AM1 & 0.0 & - & 15.1 & - \\
\hline AH2 & 0.0667 & 0.295 & 17.2 & 15 \\
AH3 & 0.0833 & 0.247 & 17.6 & 12 \\
AH4 & 0.14 & 0.174 & 18.5 & 7 \\
AH5 & 0.2 & 0.15 & 18.8 & 5
\end{tabular}

scale, $\Gamma_{\star}$ appears relatively constant, $\Gamma_{\star} \approx 0.55$ for a sizable range of Reynolds number. This value is close to that expected on the basis of modal equipartition, with one temperature mode and two independent velocity modes per wave vector in the incompressible case. The substantially smaller values of $\Gamma_{\star}$ for lower Reynolds numbers denote the fact that, at these $R e$ values, the coupling between the momentum and scalar equations becomes negligible, the temperature fluctuations become passively advected, and since the initial conditions, except for the QG runs, are equal to zero for $\theta$, the potential energy dissipation has become negligible as well. Note that these runs are performed, by necessity, at lower Reynolds numbers since the nonlinear terms are stronger in that case, unimpeded by the somewhat slower waves.

\section{The scaling behavior of magnetic helicity in Hall MHD}

We now examine some aspects of the temporal and spectral dynamics of energy and helicity in Hall-MHD, with an emphasis on the behavior of the large scales, which are scarcely studied. To that effect, we analyze data stemming from a series of numerical simulations performed at moderate resolution and in the presence of a forcing term at $k_{F} \approx 20$ (see Table 1 and equations (1-2)). These runs were already analyzed in [100] for their overall behavior, and we shall concentrate here on the properties of relative helicity.

\subsection{Temporal growth and spectral behavior}

We first give an overall vision of the temporal behavior of these flows, by examining in Fig. 4, as a function of time, the ratio $H_{C} / E_{T}$ (top left) and the kinetic helicity (top right), the relative rate of the alternate helicity $H_{X}=$ $2 H_{C}+\epsilon_{H} H_{V}$ (bottom left), and the relative rate of kinetic helicity $\sigma_{V}$ (bottom right). The color coding of the runs is given in the inset (see also Table 1), and time is expressed in units of the nonlinear turnover time. As the scale at which the Hall current becomes important increases, so does the relative amount of generalized helicity and of $H_{X}$, and the reverse is true for their 
kinetic counterpart. This is related to the fact that, as $\epsilon_{H}$ increases, kinetic helicity plays a more prominent role in the nonlinear dynamics constrained by its inviscid invariants. Contrasting with the temporal evolutions of $\sigma_{G, M, C}$ shown in [100] (see Figure 2), we see that $\sigma_{X}$ emphasizes the effect of the kinetic helicity and of the large scales as $\epsilon_{H}$ increases, but with rather similar growth rates for all runs, as seen in their slopes, displaying opposite effects of large and small scales.

This is confirmed by what we observe in Fig. 5 which gives information about the so-called relative helicity (in absolute value), $\left|H_{R}(k)\right|=\mid H_{V}(k)-$ $k^{2} H_{M}(k) \mid$, with MHD on the left and Hall-MHD on the right (note that, in $[101,102]$, one also examines, for MHD turbulence, the relative ratio of kinetic and magnetic energy and helicity). The balance between the kinetic and magnetic helicity is achieved more rapidly across scales in Hall-MHD, but at a higher level, indicating a less-efficient equipartition in Hall MHD, as expected since Alfvén waves are less prevalent. On the other hand, the gravest mode undergoes a different dynamics: the force-free field that is dominant in MHD is no longer as attractive in Hall-MHD; it is in fact replaced by a more complex Beltramization [103] (see also [104]). In the two-dimensional case in MHD, the cross-helicity is also conserved, and invariants again strongly influence the nonlinear dynamics. For example, a recent numerical analysis at high resolution shows that small-scale current and vorticity structures, although highly intermittent, are organized in a manner compatible with variational principles based on these invariants [105]. 


\subsection{Scaling with Hall parameter}

We finish this short review by recalling what scaling is obtained, in terms of $\epsilon_{H}$, in measuring the strength of the inverse helicity cascades as the Hall current becomes more important in the large scales. To this end, in Fig. 6, we give the slope of the temporal growth of the ratio of magnetic to kinetic energy as a function of the Hall control parameter $\epsilon_{H}$, in lin-log coordinates. An exponential decay scaling emerges, with a slope of $\approx 12.7$, which can be shown to be compatible with the scaling found in [100] for the magnetic and generalized helicity, $H_{M}$ and $H_{G}$.

As stated before, one particularity of Hall MHD is that $H_{G}$ involves various scales : when $\epsilon_{H}$ increases, smaller scales get involved in the constraining dynamics of maintaining invariants because of detailed conservation for each triadic modal interaction. Thus, the inverse cascade of magnetic helicity is less efficient and there is less growth of the magnetic energy at large scale, with a resulting declining ratio.

The physical argument that leads to such an exponential decrease with $\epsilon_{H}$ is based on the scaling of the generalized helicity and of the magnetic helicity $H_{G, M}(k) \sim k^{-2}$, together with the plausible assumptions of maximal magnetic helicity in the inverse cascade and of kinetic and magnetic energy equipartition due to Alfvén waves, in an intermediate regime of parameters. Of course, these results may depend on Reynolds number (and on numerical resolution as well), and in particular on whether or not resolving the small-scale inertial range will affect the large-scale growth of magnetic helicity at fixed $\epsilon_{H}$. This was not the case for the inverse cascade of magnetic helicity in MHD once the Reynolds number at the forcing scale was of order unity or more, but this point remains to be studied in the future for Hall-MHD.

We finally note that the non-local properties of the inverse cascade of magnetic helicity for Hall-MHD have been studied very recently in the framework of a two-fluid model [106]. These authors conclude that the system is strongly sensitive to the value of the governing parameter (the ratio of the wave period to the eddy turn-over time).

\section{Conclusion}

In the ideal (non-dissipative) case, it is known that the role of invariants in the dynamical behavior of nonlinear multi-dimensional systems is essential, as discussed in earlier sections. This role carries over to the dissipative (turbulent) case, and it leads to the breaking of the universality of scaling laws [107]. Invariants are physically important, be it only because of the property of detailed conservation: any nonlinear exchange between three (Fourier) modes satisfying the convolution compatibility condition is conservative of the invariants, such as energy or helicity. This translates itself, in configuration space, in a detailed conservation embodied in the so-called exact laws of turbulence strongly constraining the scaling of interactions of third-order structure func- 


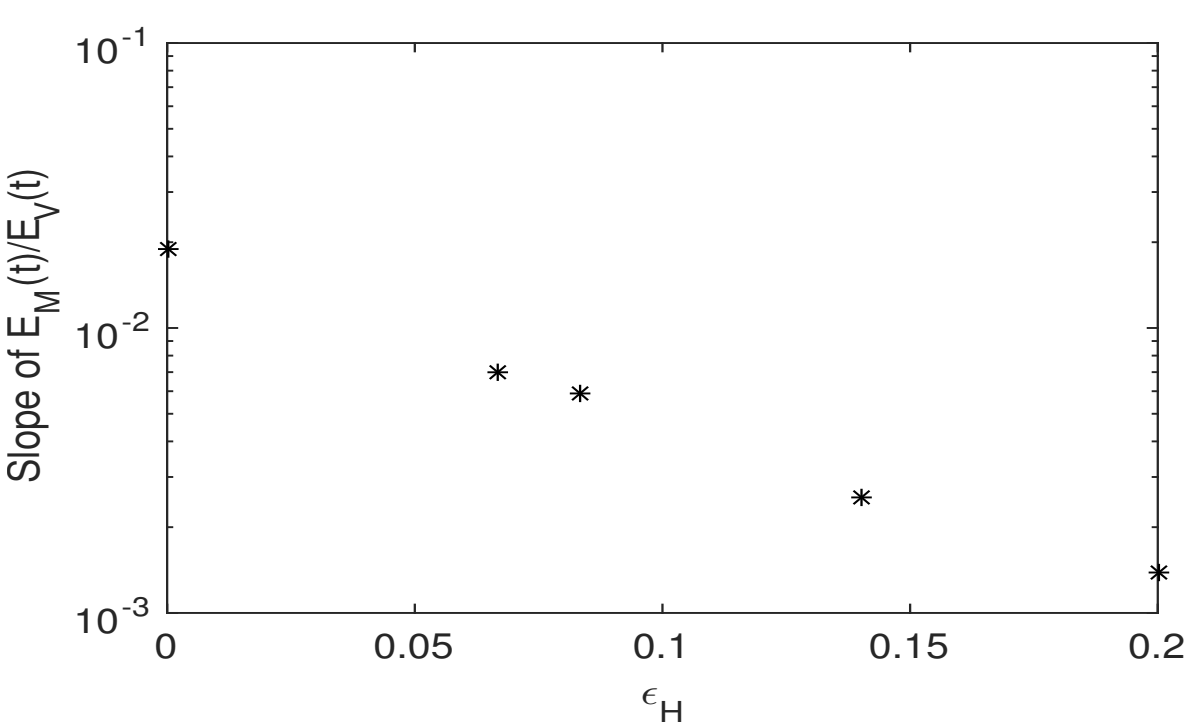

Fig. 6 Slope of the temporal decay of the magnetic to kinetic energy ratio as a function of the Hall control parameter $\epsilon_{H}$. Note the exponential scaling with $\epsilon_{H}$ [100].

tions over a distance $r$ : in fact, a linear variation in $r$ is obtained with a known coefficient of proportionality $[108,66,68]$.

Beyond magnetic helicity, a second quadratic invariant has been known to exist for a long time [109], namely the cross-correlation $H_{C}=\langle\mathbf{v} \cdot \mathbf{b}\rangle$. It was shown in [110], using a second-order turbulence closure, that its dynamical evolution is towards a one-signed correlation in the inertial range with a change of sign at the dissipation scale, together with an inertial spectral index that changes with the relative rate of cross-helicity $\sigma_{C}$. These results were later confirmed by direct numerical simulations in two space dimensions [111] (see also [112] in three dimensions). Symmetries, as embodied for example in a strong $\sigma_{C}$ or $\sigma_{M}$, or a weak $\sigma_{V}$ for a non-helical forcing flow, can impede dynamo action (see [113] for the specific case of the so-called Taylor-Green vortex with $\left.\sigma_{V}=0\right)$. It is also known that $H_{C}$ plays a role in the dynamo problem of generation of magnetic field through vortex stretching [114], including in the Hall-MHD case [104], and on the rate of the turbulent cascade to small scales as observed in the solar wind [115,116] (see [117] for a recent observation in the context of the newly-launched Parker Solar Probe).

New studies continue to deal with this issue. In [118], it is shown that, in the forced case, there is an enhanced inverse transfer of kinetic energy to the large scales. The role of a (generalized) cross-helicity is studied in [119] (see also [120]). It can be seen as allowing for a smooth transition from MHD scales to kinetic scales. In these papers, it is claimed in particular that, using a weak turbulence approach, the forcing may alter the dynamics in the presence of dispersive waves. Furthermore, these authors can argue, on the basis of a model based on nonlinear diffusion equations, that different streams ob- 
served in highly Alfvénic regions of the solar wind with differing cross-helicity amounts (or for imbalanced states), are mixed. Finally, let us mention the discovery of changes of signs of the cross-helicity observed, thanks again to the Parker Solar Probe, in so-called magnetic switchbacks [121], that exhibit polarity reversals.

There is no doubt that the tremendous efforts of the international community behind the many successful space missions, reaching from fluid scales to the kinetic scales, in parallel with numerical efforts in the context of HighPerformance Computing and the development of algorithms using Artificial Intelligence, will further bring more detailed understanding of the prevailing interactions in these complex plasma flows.

\section{Declarations:}

Funding: The work of Julia E. Stawarz was supported in part by the project UKR/STFC grant ST/S000364/1.

Conflicts of interest/Competing interests: The authors declare that there are no conflicts of interest.

Availability of data and material and code: The authors declare that the calculation material and the code are all available upon request.

Authors' contributions: All three authors have equally contributed to the research work presented in this article. Finally the authors declare that this manuscript is in compliance with the Journal Ethical Standards.

Acknowledgements This text emanates from the talk given by one of us (AP) at the meeting held in honor of PierLuigi Veltri in October 2019 at the University of Calabria in Cosenza (Italy). It is a pleasure to acknowledge the many facets of his work, a little part of which is evoked here in the context of specific Solar Wind theoretical models and observations, and to reminisce about our discussions on many issues of MHD turbulence and on the complexity of the behavior of such systems. The runs analyzed in this paper have used an open allocation on the Janus super-computer at LASP/CU, which is gratefully acknowledged. NCAR is supported by the National Science Foundation. Support for AP, from LASP and in particular from Bob Ergun, is gratefully acknowledged as well.

\section{References}

1. A. Alexakis, Astrophys. J. 667, L93 (2007)

2. P.D. Mininni, A. Alexakis, A. Pouquet, J. Plasma Phys. 73, 377 (2007)

3. P. Mininni, A. Alexakis, A. Pouquet, Phys. Rev. E 74, 016303 (2006)

4. T. Passot, P. Sulem, Astrophys. J. Lett. 812(L37) (2015)

5. P. Veltri, Plasma Phys. Control. Fusion 41, A787 (1999)

6. P. Veltri, V. Carbone, F. Lepreti, G. Nigro, Encyclopedia of Complexity and System Science R.A. Meyers Ed., Springer (2009)

7. W. Matthaeus, M. Velli, Space Sci. Rev. 160, 145 (2011)

8. S. Galtier, Introduction to Modern Magnetohydrodynamics (Cambridge University Press, 2016)

9. R. Bruno, V. Carbone, Living Rev. Solar Phys. 2, 4 (2005)

10. W.H. Matthaeus, M. Wan, S. Servidio, A. Greco, K.T. Osman, S. Oughton, P. Dmitruk, Phil. Trans. R. Soc. A 373(20140154) (2015) 
11. R.E. Ergun, K.A. Goodrich, F.D. Wilder, N. Ahmadi, J.C. Holmes, S. Eriksson, J.E. Stawarz, R. Nakamura, K.J. Genestreti, M. Hesse, J.L. Burch, R.B. Torbert, T.D. Phan, S.J. Schwartz, J.P. Eastwood, R.J. Strangeway, O. Le Contel, C.T. Russell, M. Argall, P.A. Lindqvist, L.J. Chen, P.A. Cassak, B.L. Giles, J.C. Dorelli, D. Gershman, T.W. Leonard, B. Lavraud, A. Retino, W. Matthaeus, A. Vaivads, Geophys. Res. Lett. 45, 3338 (2018)

12. O. Le Contel, A. Retinó, H. Breuillard, L. Mirioni, P. Robert, A. Chasapis, B. Lavraud, T. Chust, L. Rezeau, F.D. Wilder, D.B. Graham, M.R. Argall, D.J. Gershman, P.A. Lindqvist, Y.V. Khotyaintsev, G. Marklund, R.E. Ergun, K.A.G. andJ. L. Burch, R.B. Torbert, J. Needell, M. Chutter, D. Rau, I. Dors, C.T. Russell, W. Magnes, R.J. Strangeway, K.R. Bromund, H.K. Leinweber, F. Plaschke, D. Fischer, B.J. Anderson, G. Le, T.E. Moore, C.J. Pollock, B.L. Giles, J.C. Dorelli, L. Avanov, Y. Saito, Geophys. Res. Lett. 43, 5943 (2016)

13. A. Chasapis, W.H. Matthaeus, T.N. Parashar, M. Wan, C.C. Haggerty, C.J. Pollock, B.L. Giles, W.R. Paterson, J. Dorelli, D.J. Gershman, R.B. Torbert, C.T. Russell, P.A. Lindqvist, Y. Khotyaintsev, T.E. Moore, R.E. Ergun, J.L. Burch, Astrophys. J. Lett. 856, L19 (2018)

14. T.D. Phan, J.P. Eastwood, M.A. Shay, J.F. Drake, B.U.Ö. Sonnerup, M. Fujimoto, P.A. Cassak, M. Øieroset, J.L. Burch, R.B. Torbert, A.C. Rager, J.C. Dorelli, D.J. Gershman, C. Pollock, P.S. Pyakurel, C.C. Haggerty, Y. Khotyaintsev, B. Lavraud, Y. Saito, M. Oka, R.E. Ergun, A. Retino, O. Le Contel, M.R. Argall, B.L. Giles, T.E. Moore, F.D. Wilder, R.J. Strangeway, C.T. Russell, P.A. Lindqvist, W. Magnes, Nature 557, 202 (2018)

15. J.E. Stawarz, D.J. Gershman, J.P. Eastwood, T.D. Phan, I.L. Gingell, M.A. Shay, J.L. Burch, R.E. Ergun, B.L. Giles, O. Le Contel, P.A. Lindqvist, C.T. Russell, R.J. Strangeway, R.B. Torbert, M.R. Argall, D. Fischer, W. Magnes, L. Franci, Astrophys. J. Lett. 877, L37(7 pp) (2019)

16. P.S. Iroshnikov, Sov. Astron. 7, 566 (1963)

17. R.H. Kraichnan, Phys. Fluids 8, 1385 (1965)

18. M. Dobrowolny, A. Mangeney, P. Veltri, Phys. Rev. Lett. 45, 144 (1980)

19. L. Sorriso-Valvo, R. Marino, V. Carbone, A. Noullez, F. Lepreti, P. Veltri, R. Bruno, B. Bavassano, E. Pietropaolo, Phys. Rev. Lett. 99(115001) (2007)

20. R. Marino, L. Sorriso-Valvo, V. Carbone, P. Veltri, A. Noullez, R. Bruno, Planetary and Space Sc. 59, 592 (2011)

21. L. Sorriso-Valvo, V. Carbone, P. Giuliani, P. Veltri, R. Bruno, V. Antoni, E. Martines, Planet. Space Sci. 49, 1193 (2001)

22. P. Veltri, G. Nigro, F. Malara, V. Carbone, A. Mangeney, Nonlinear Proc. Geophys 12, $245(2005)$

23. A. Fujisawa, A. Shimizu, H. Nakano, S. Ohshima, K. Itoh, Y. Nagashima, S.I. Itoh, H. Iguchi, Y. Yoshimura, T. Minami, K. Nagaoka, C. Takahashi, M. Kojima, S. Nishimura, M. Isobe, C. Suzuki, T. Akiyama, T. Ido, K. Matsuoka, S. Okamura, P.H. Diamond, Plasma Physics and Controlled Fusion 49(3), 211 (2007). URL http://stacks.iop.org/0741-3335/49/i=3/a =002

24. V. Abramenko, V. Yurchyshyn, Astrophys. J. 722, 122 (2010)

25. K.T. Osman, W.H. Matthaeus, J.T. Gosling, A. Greco, S. Servidio, B. Hnat, S.C. Chapman, T.D. Phan, Phys. Rev. Lett. 112, 215002 (2014)

26. L. Sorriso-Valvo, R. Marino, L. Lijoi, S. Perri, V. Carbone, Astrophys. J. 807, 86 (2015)

27. L. Sorriso-Valvo, F. Catapano, A. Retinò, L. Olivier, D. Perrone, O.W. Roberts, J.T. Coburn, V. Panebianco, F. Valentini, S. Perri, A. Greco, F. Malara, V. Carbone, P. Veltri, O. Pezzi, F. Fraternale, F. Di Mare, R. Marino, B. Giles, T.E. Moore, C.T. Russell, R.B. Torbert, J.L. Burch, Y.V. Khotyaintsev, Phys. Rev. Lett. 122, 035102 (2019)

28. P. Mininni, A. Pouquet, Phys. Rev. E 80, 025401 (2009)

29. R. Grauer, H. Homann, J.F. cois Pinton, New J. Phys. 14, 063016 (2012)

30. H. Karimabadi, V. Roytershteyn, M. Wan, W.H. Matthaeus, W. Daughton, P. Wu, M. Shay, B. Loring, J. Borovsky, E. Leonardis, S.C. Chapman, T.K.M. Nakamura, Phys. Plasmas 20, 012303 (2013) 
31. H. Homann, Y. Ponty, G. Krstulovic, R. Grauer, New J. Phys. 16, 075014 (2014)

32. R. Meyrand, K.H. Kiyani, S. Galtier, J. Fluid Mech. 770, R1, 1 (2015)

33. E.T. Lu, R.J. Hamilton, Astrophys. J. 380, L89 (1991)

34. G. Boffetta, V. Carbone, P. Giuliani, P. Veltri, A. Vulpiani, Phys. Rev. Lett. 83, 4662 (1999)

35. V. Uritsky, M. Paczuski, J.M. Davila, S.I. Jones, Phys. Rev. Lett. 99, 025001 (2007)

36. A.J. Klimas, V.M. Uritsky, Phys. Rev. E 95, 023209 (2017)

37. E. Buchlin, V. Aletti, S. Galtier, M. Velli, G. Einaudi, J.C. Vial, Astron. Astrophys. 406, 1061 (2003)

38. W. Matthaeus, S. Lamkin, Phys. Fluids 29, 2513 (1986)

39. H. Politano, A. Pouquet, P. Sulem, Phys. Fluids B 1, 2330 (1989)

40. H. Politano, A. Pouquet, P. Sulem, Phys. Plasmas 2, 2931 (1995)

41. Y. Zhou, W. Matthaeus, P. Dmitruk, Rev. Mod. Phys. 76, 1015 (2004)

42. R. Marino, L. Sorriso-Valvo, R. D'Amicis, V. Carbone, R. Bruno, P. Veltri, Astrophys. J. 750, 41 (2012)

43. N.F. Loureiro, A.A. Schekochihin, D.A. Uzdensky, Phys. Rev. E 87, 013102 (2013)

44. V. Zhdankin, S. Boldyrev, D.A. Uzdensky, Phys. Plasmas 23(5), 055705 (2016)

45. R. Chhiber, M. Goldstein, B.A. Maruca, A. Chasapis, W.H. Matthaeus, M. Ruffolo, R. Bandyopadhyay, T.N. Parashar, R. Qudsi, T.D. de Wit, S. Bale, J. Bonnell, K. Goetz, P. Harvey, R. MacDowall, D. Malaspina, M. Pulupa, J. Kasper, K. Korreck, A. Case, M. Stevens, P. Whittlesey, D. Larson, R. Livi, M. Velli, N. Raouafi, Astrophys. J. Supp. 246(31) (2020)

46. G. Ivey, K. Winters, J. Koseff, Ann. Rev. Fluid Mech. 40, 169 (2008)

47. J. Sun, C.J. Nappo, L. Mahrt, D. Belusic, J.J. Finnigan, C. Yagüe, B. Grisogono, A. Pouquet, D.R. Stauer, R.B. Smith, G. Svenson, M. Pulido, C. Staquet, S.D. Mayor, Q. Jiang, B. Galperin, A.A. Grachev, W.D. Neff, Rev. Geophys. 53, doi:10.1002/2015RG000487, 1 (2015)

48. D. Rosenberg, P.D. Mininni, R. Reddy, A. Pouquet, Atmosphere 11, 178 (2020)

49. M. Fontana, O.P. Bruno, P.D. Mininni, P. Dmitruk, Comp. Phys. Comm. 256, 107482 (2020)

50. R. Hide, Geophys. Astrophys. Fluid Dyn. 7, 157 (1976)

51. R. Hide, Q. J. Roy. Met. Soc. 128, 1759 (2002)

52. R. Marino, P. Mininni, D. Rosenberg, A. Pouquet, Phys. Rev. E 87, 033016 (2013)

53. A. Pouquet, D. Rosenberg, R. Marino, Phys. Fluids 31, 105116 (2019)

54. R. Scott, F. Wang, J. Phys. Oceano. 35, 1650 (2005)

55. D. Balwalda, J. LaCasce, K. Speer, Geophys. Res. Lett. 43, 10,856 (2016)

56. C. Morize, F. Moisy, M. Rabaud, Phys. Fluids 17(095105) (2005)

57. P. Mininni, A. Pouquet, Phys Rev E 79, 026304 (2009)

58. R. Marino, A. Pouquet, D. Rosenberg, Phys. Rev. Lett. 114, 114504 (2015)

59. H. Kafiabad, P. Bartello, J. Fluid Mech. 795, 914 (2016)

60. A. Alexakis, L. Biferale, Physics Reports 762, 1 (2018)

61. A. van Kan, T. Nemoto, A. Alexakis, J. Fluid Mech. 878, 356 (2019)

62. K. Seshasanayan, S. Benavides, A. Alexakis, Phys. Rev. E 90, 051003(R) (2014)

63. K. Seshasanayan, A. Alexakis, Phys. Rev. E 93, 013104 (2016)

64. R.M.B. Young, P.L. Read, Nature Phys. 13, 1135 (2017)

65. H. Politano, A. Pouquet, Phys. Rev. E 57, R21 (1998)

66. H. Politano, A. Pouquet, Geophys. Res. Lett. 25, 273 (1998)

67. H. Politano, T. Gomez, A. Pouquet, Phys. Rev. E 68, 026315 (2003)

68. S. Galtier, J. Phys. A.: Math. Theor. 51(293001) (2018)

69. R. Marino, P. Mininni, D. Rosenberg, A. Pouquet, EuroPhys. Lett. 102, 44006 (2013)

70. A. Pouquet, D. Rosenberg, R. Marino, C. Herbert, J. Fluid Mech. 844, 519 (2018)

71. R. Benzi, S. Ciliberto, R. Tripiccione, C. Baudet, F. Massaioli, S. Succi, Phys. Rev. E 48, R29 (1993)

72. U. Frisch, Pouquet, P. Sulem, M. Meneguzzi, J. Méc. Théor. Appl. 2, Special Issue, $191(1983)$

73. M.E. Brachet, M. Bustamante, G. Krstulovic, P. Mininni, A. Pouquet, D. Rosenberg, Phys. Rev. E 87, 013110 (2013)

74. C. Cichowlas, P. Bonaïti, F. Debbasch, M. Brachet, Phys Rev. Lett. 95(264502) (2005) 
75. G. Krstulovic, M. Brachet, A. Pouquet, Phys. Rev. E 84, 016410 (2011)

76. L. Sorriso-Valvo, V. Carbone, V. Abramenko, V. Yurchysshyn, A. Noullez, H. Politano A. Pouquet, P. Veltri, Planet. Space Sc. 52, 937 (2004)

77. M. Øieroset, R. Lin, T. Phan, D.E. Larson, S. Bale, Phys. Rev. Lett. 89, 195001 (2002)

78. J.F. Drake, M. Swisdak, H. Che, M.A. Shay, Nature 443 (2006)

79. D. Rosenberg, A. Pouquet, R. Marino, P. Mininni, Phys. Fluids 27, 055105 (2015)

80. C. Rorai, P. Mininni, A. Pouquet, Phys. Rev. E 89, 043002 (2014)

81. F. Feraco, R. Marino, A. Pumir, L. Primavera, P. Mininni, A. Pouquet, D. Rosenberg, Eur. Phys. Lett. 123, 44002 (2018)

82. D. Buaria, A. Pumir, F. Feraco, R. Marino, A. Pouquet, D. Rosenberg, L. Primavera, Phys. Rev. Fluids 5, 064801 (2020)

83. A. Pumir, Phys. Fluids 8, 3112 (1996)

84. Y. Narita, K.H. Glassmeier, R. Treumann, Phys. Rev. Lett. 97, 191101 (2006)

85. M.S. Paoletti, M.E. Fisher, K.R. Sreenivasan, D.P. Lathrop, Phys. Rev. Lett. 101, $154501(2008)$

86. V. Petoukhov, A. Eliseev, R. Klein, H. Oesterle, Tellus 60A, 11 (2008)

87. P. Vieillefosse, Physica A 125, 150 (1984)

88. N. Sujovolsky, P. Mininni, Phys. Rev. Fluids 4, 052402 (2019)

89. N. Sujovolsky, P. Mininni, ArXiv:1912.03160v1 (2020)

90. M.S. Chong, A.E. Perry, B.J. Cantwell, Phys. Fluids A 2(5), 765 (1990). DOI $10.1063 / 1.857730$

91. P.D. Mininni, A. Pouquet, Phys. Rev. Lett. 99, 254502 (2007)

92. L. Djenidi, N. Lefeuvre, M. Kamruzzaman, R. Antonia, J. Fluid Mech. 817, 61 (2017)

93. S.R. Yoffe, W.D. McComb, Phys. Rev. F 3, 104605 (2018)

94. R. Bandyopadhyay, S. Oughton, M. Wan, W.H. Matthaeus, R. Chhiber, T.N. Parashar, Phys. Rev. X 8(041052) (2018)

95. G. Howes, J. TenBarge, W. Dorland, Phys. Plasmas 18, 102305 (2011)

96. A. Schreiner, J. Saur, Astrophysical J. 835, 133 (2017)

97. E. Dewan, J. Geophys. Res. 102, 29,799 (1997)

98. R. Marino, P. Mininni, D. Rosenberg, A. Pouquet, Phys. Rev. E 90, 023018 (2014)

99. D. Rosenberg, R. Marino, C. Herbert, A. Pouquet, Eur. Phys. J. E 39, 8 (2016)

100. A. Pouquet, J. Stawarz, D. Rosenberg, Atmosphere 11, 00203 (2020)

101. W. Müller, S. Malapaka, A. Busse, Phys. Rev. E 85, 015302 (2012)

102. W. Müller, S. Malapaka, Geophys. Astrophys. Fluid Dyn. 107, 93 (2013)

103. S. Mahajan, Z. Yoshida, Phys. Rev. Lett. 99, 4863 (1998)

104. P. Mininni, D.O. Gómez, S.M. Mahajan, Astrophys. J. 567, L81 (2002)

105. A. de Giorgio, S. Servidio, P.L. Veltri, Scientific Reports 7, 13849 (2017)

106. G. Miloshevich, D. Laveder, T. Passot, P. Sulem, Preprint, see arXiv:2007.06976v1 (2020)

107. P. Mininni, D. Rosenberg, A. Pouquet, J. Fluid Mech. 699, 263 (2012)

108. A.N. Kolmogorov, Dokl. Akad. Nauk SSSR 30, 9 (1941)

109. L. Woltjer, Rev. Mod. Phys. 32, 914 (1960)

110. R. Grappin, U. Frisch, J. Léorat, A. Pouquet, Astron. Astrophys. 102, 6 (1982)

111. A. Pouquet, P.L. Sulem, M. Meneguzzi, Phys. Fluids 31, 2635 (1988)

112. A. Beresniak, Astrophys. J. Lett. 784(L20) (2014)

113. C. Nore, M. Brachet, H. Politano, A. Pouquet, Phys. Plasmas Lett. 4, 1 (1997)

114. N. Yokoi, Geophys. Astrophys. Fluid Dyn. 107, 114 (2013)

115. C.W. Smith, J. Stawarz, B.J. Vasquez, M.A. Forman, B.T. MacBride, Phys. Rev. Lett. 103, $201101(2009)$

116. J. Stawarz, C. Smith, B. Vasquez, M. Forman, B.T.MacBride, Astrophys. J. 713, 920 (2010)

117. T.N. Parashar, M. Goldstein, B.A. Maruca, W.H. Matthaeus, M. Ruffolo, R. Bandyopadhyay, R. Chhiber, A. Chasapis, R. Qudsi, D. Vech, D.A. Roberts, S.D. Bale, J.W. Bonnell, R.J. MacDowall, D. Malaspina, M. Pulupa, J.C. Kasper, P. Whittlesey, D. Larson, R. Livi, M. Velli, N. Raouafi, Astrophys. J. Supp. 246, 58 (2020)

118. A. Brandenburg, S. Oughton, Astron. Nachrichten 339, 641 (2018)

119. T. Passot, P.L. Sulem, J. Plasma Phys. 85, 905850301 (2019)

120. G. Miloshevich, T. Passot, P. Sulem, Astrophys. J. Lett. 888, L7 (2020)

121. M.D. McManus, T.A. Bowen, A. Mallet, C.H. Chen, B.D. Chandran, S.D. Bale, R. Livi, D.E. Larson, T.D. de Wit, J. Kasper, M. Stevens, P. Whittlesey, K.E. Korreck, K. Goetz, P.R. Harvey, M. Pulupa, R.J. MacDowal, D.M. Malaspina, A.W. Case, J. Bonnell, Astrophys. J. Suppl. Series 246, 67 (2020) 




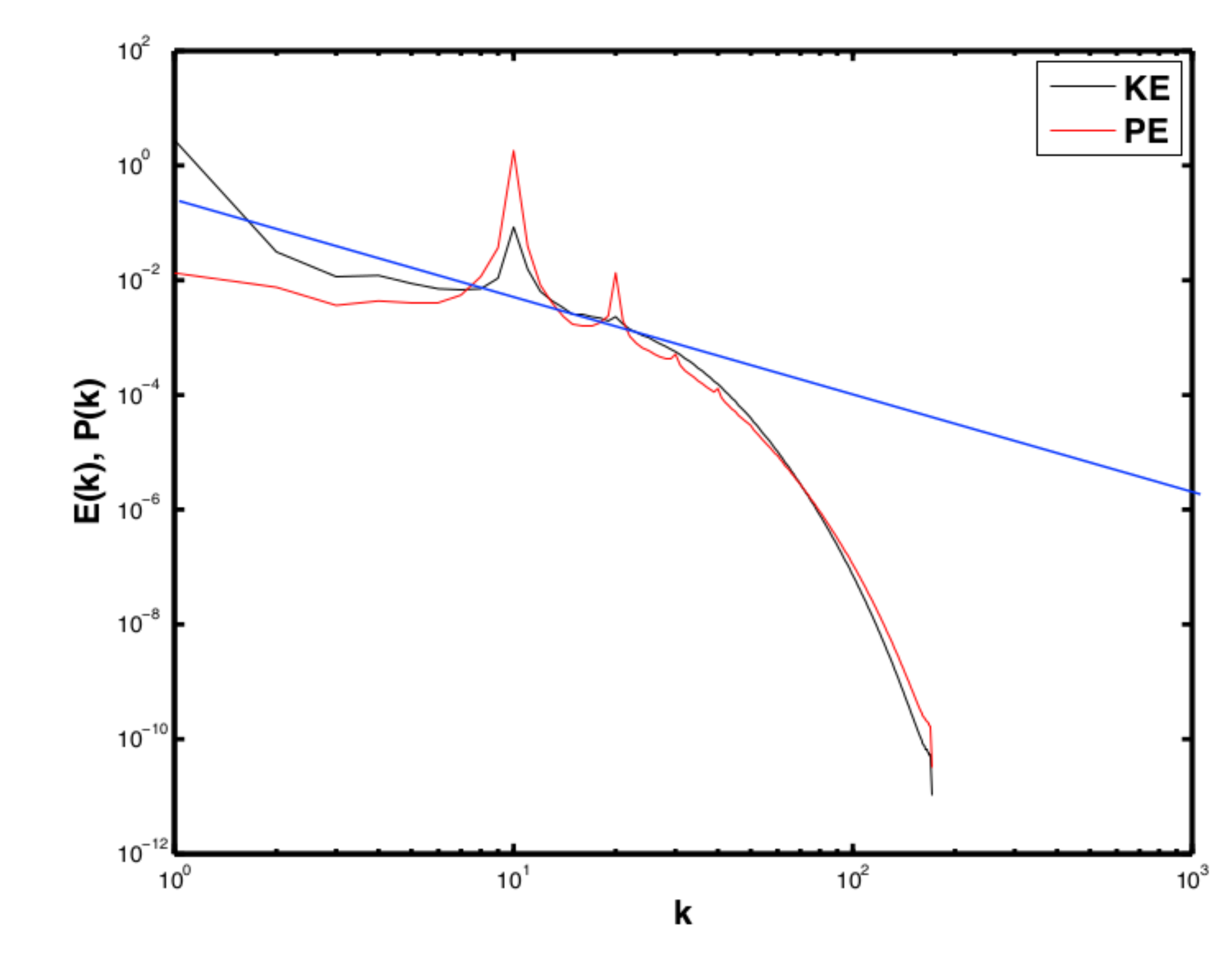

$\begin{array}{ll}\text { Figure } 1 \text { top right } & \begin{array}{l}\text { Click here to access/download;Figure;pouquet-figure1- } \\ \text { topright.pdf }\end{array}\end{array}$

$\underline{\underline{ \pm}}$

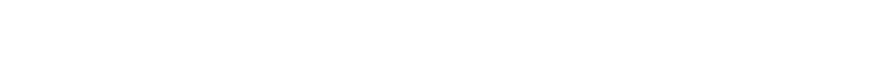
(1)

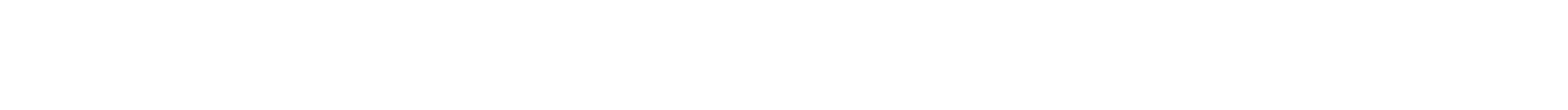
( topright.pdf 

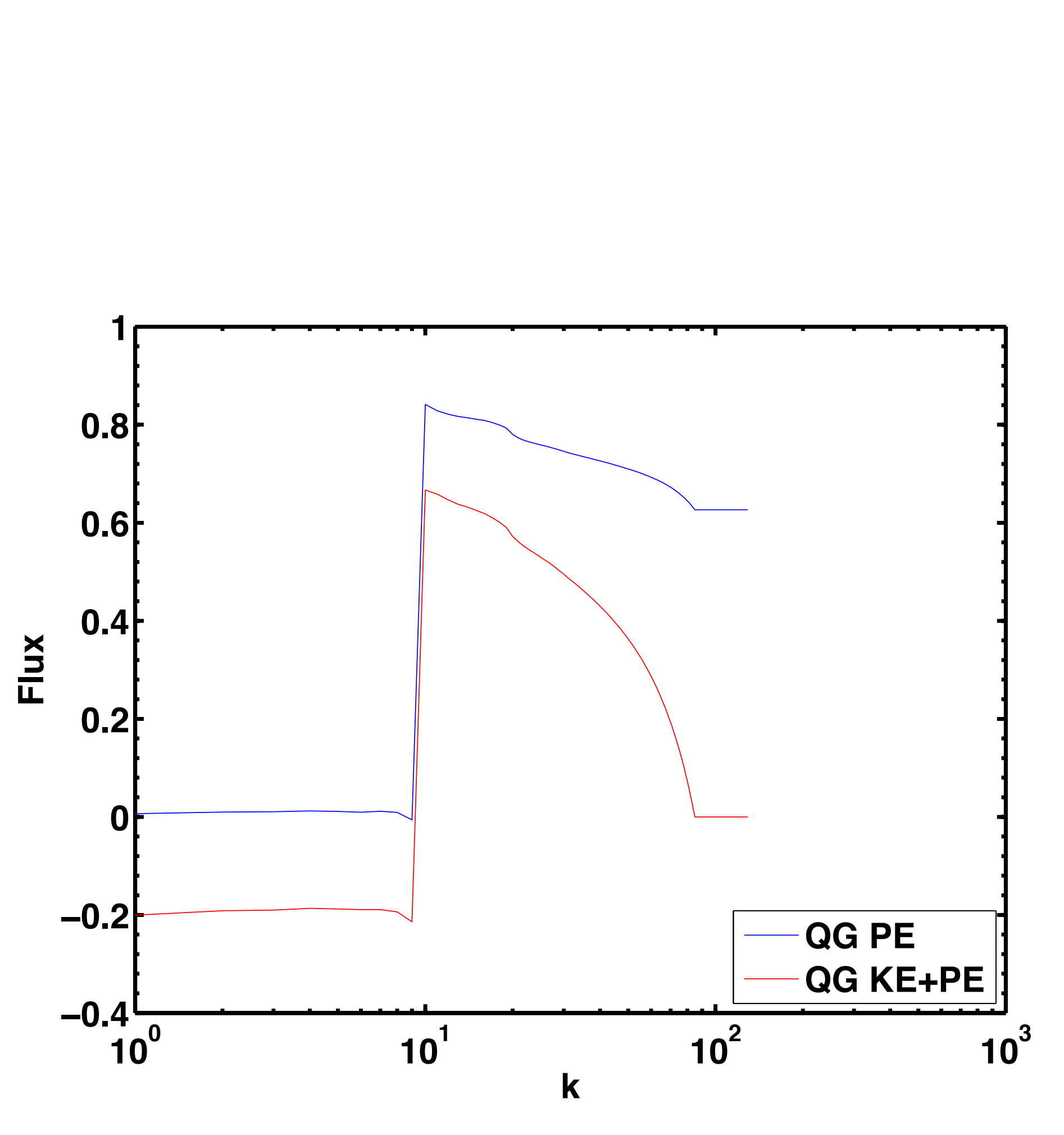

\begin{abstract}
(a)
\end{abstract}
(1)

(1)

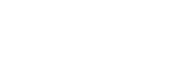

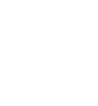

\title{
k
}




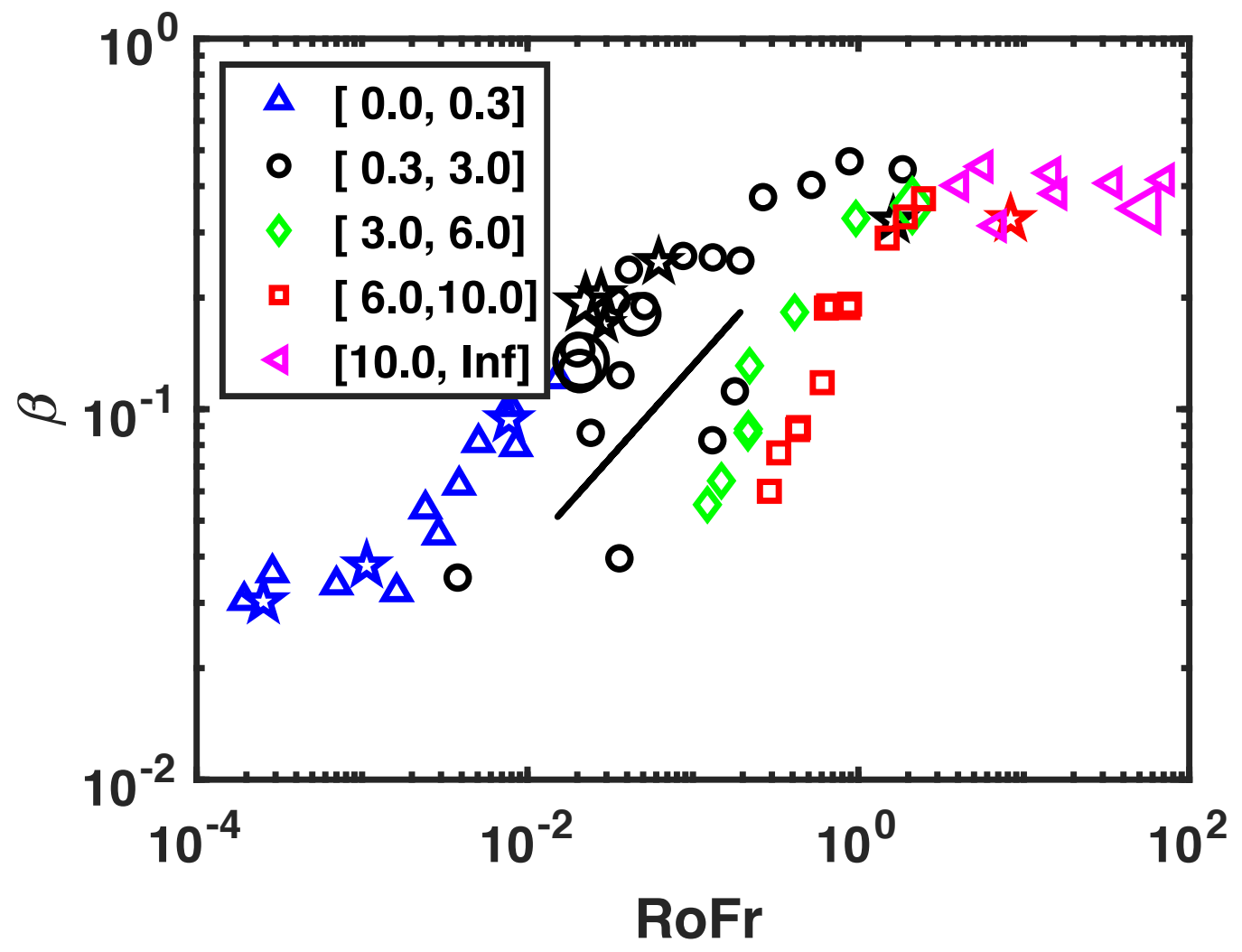




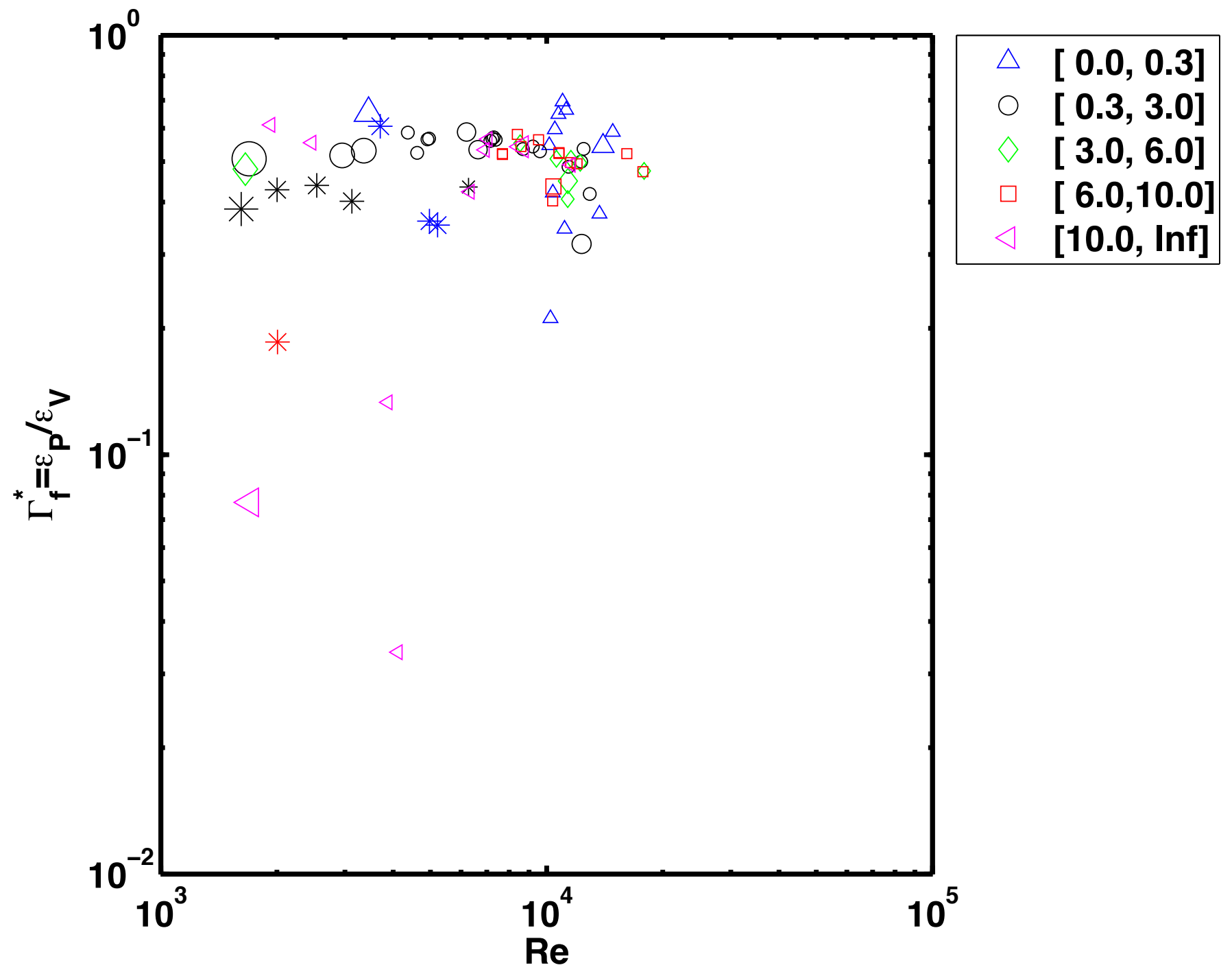




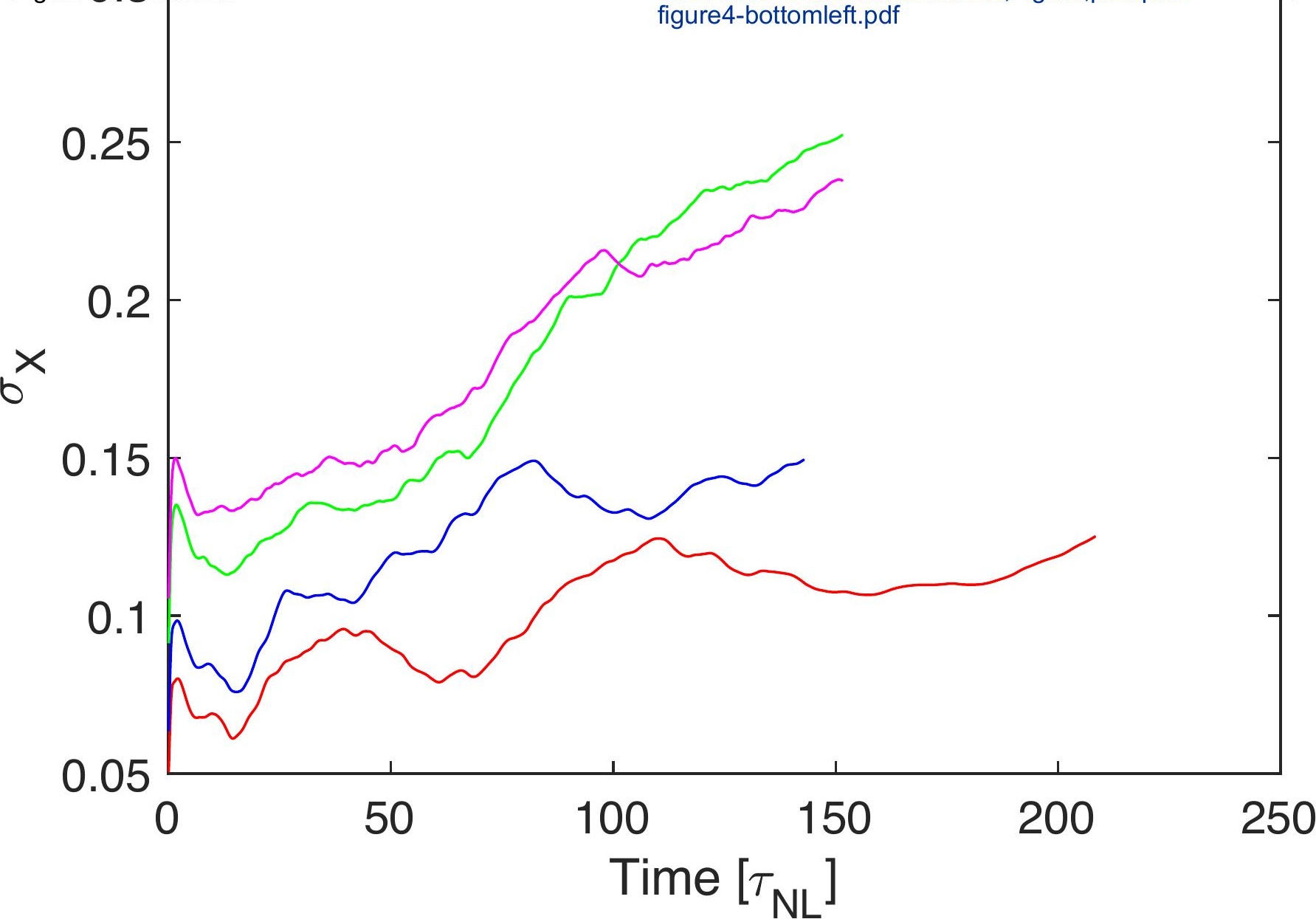




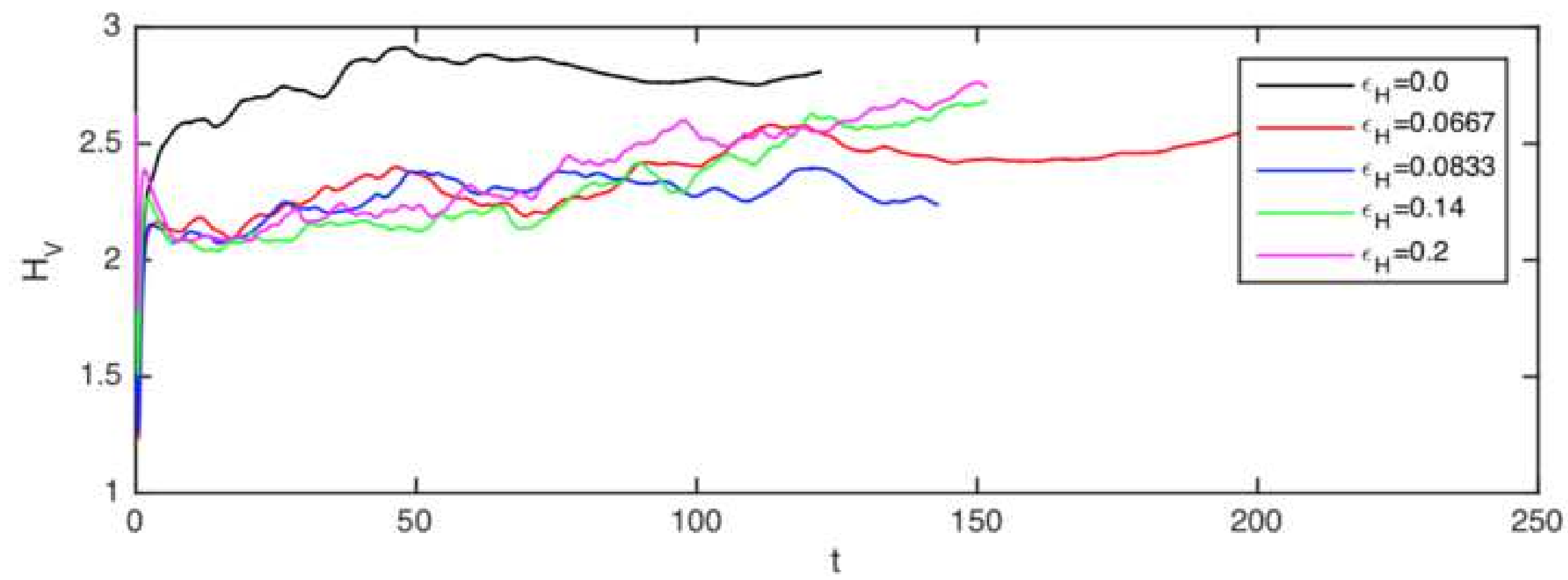




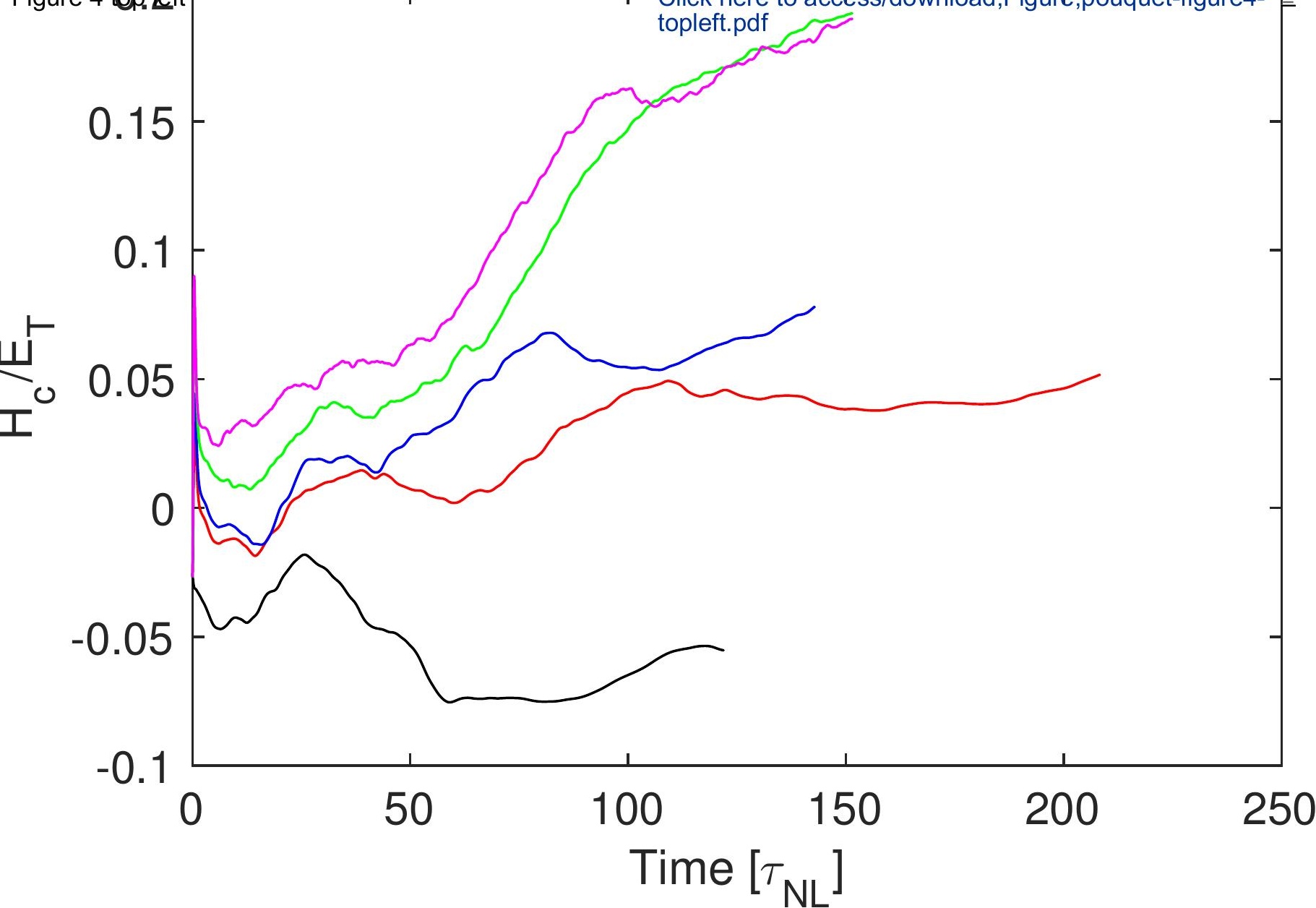




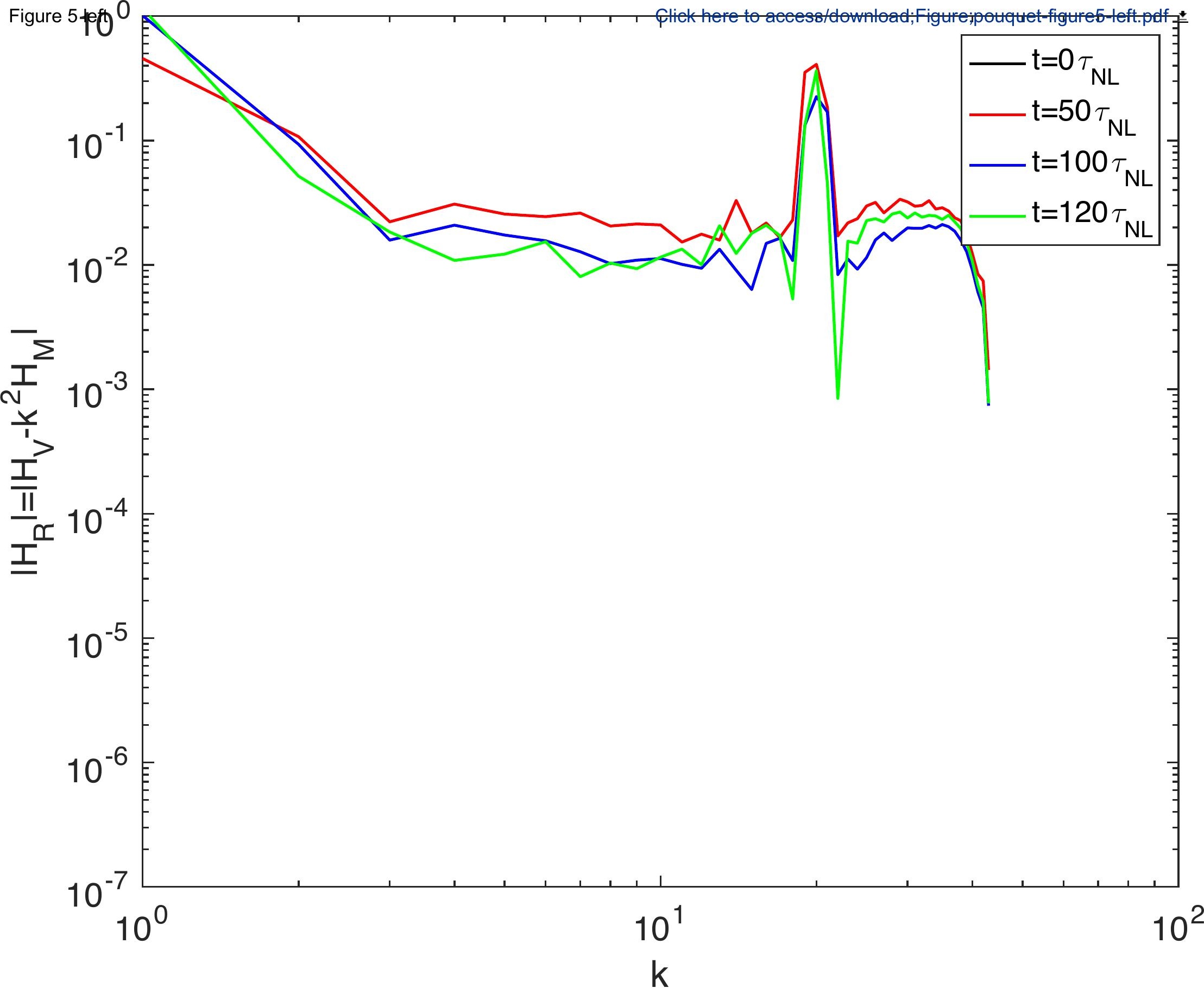




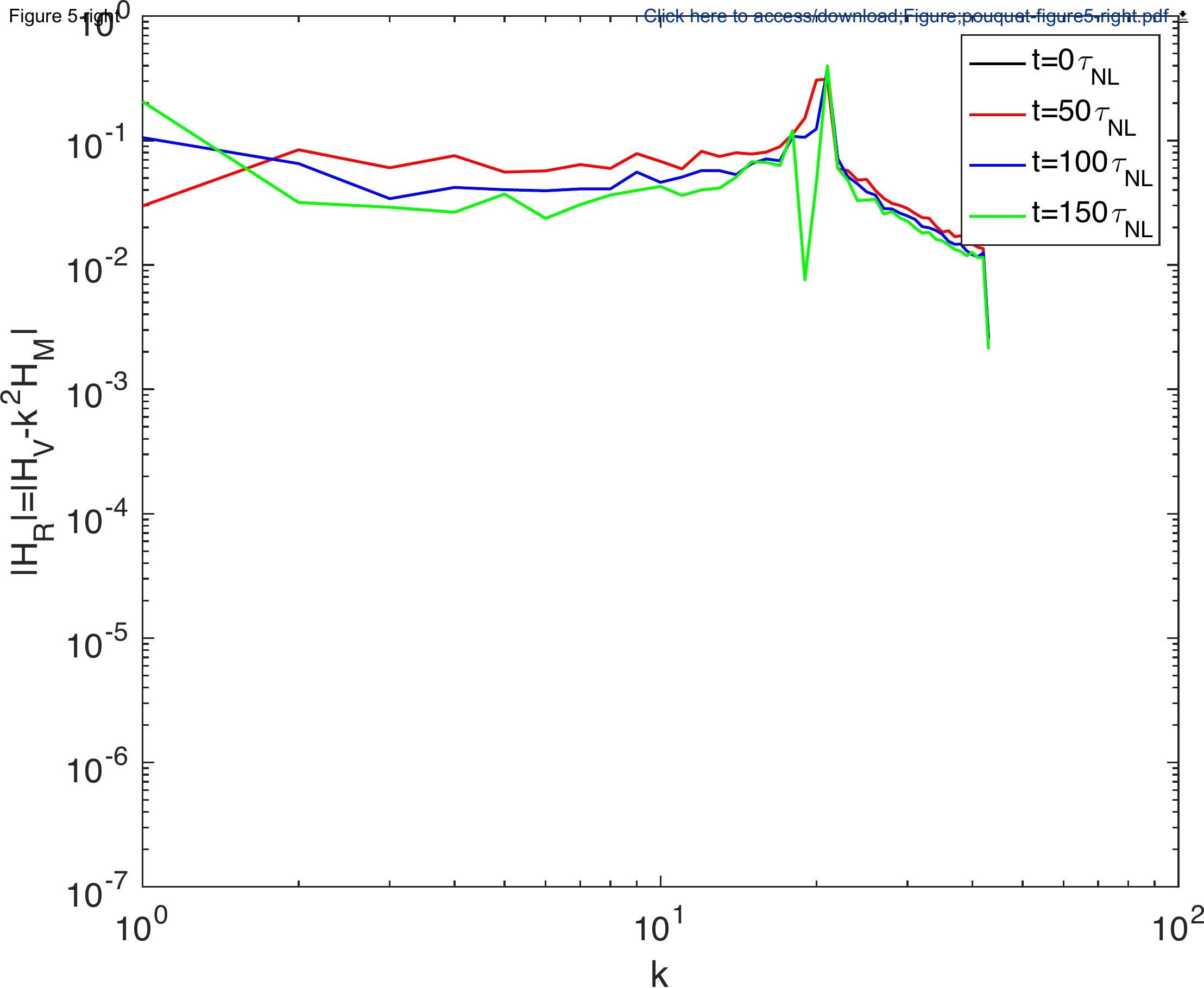


$10^{-3}$

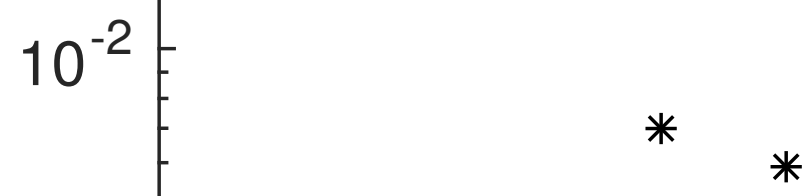
0
0.05
0.1
$\epsilon_{\mathrm{H}}$

0.15

0.2 